\title{
Experimental Evaluation of Acoustic Engine Liner Models Developed with COMSOL Multiphysics
}

\author{
Noah H. Schiller ${ }^{1}$ and Michael G. Jones ${ }^{2}$ \\ NASA Langley Research Center, Hampton, VA 23681 \\ Brandon Bertolucci ${ }^{3}$ \\ The Boeing Company, Seattle, WA 98124
}

\begin{abstract}
Accurate modeling tools are needed to design new engine liners capable of reducing aircraft noise. The purpose of this study is to determine if a commercially-available finite element package, COMSOL Multiphysics, can be used to accurately model a range of different acoustic engine liner designs, and in the process, collect and document a benchmark dataset that can be used in both current and future code evaluation activities. To achieve these goals, a variety of liner samples, ranging from conventional perforate-over-honeycomb to extendedreaction designs, were installed in one wall of the grazing flow impedance tube at the NASA Langley Research Center. The liners were exposed to high sound pressure levels and grazing flow, and the effect of the liner on the sound field in the flow duct was measured. These measurements were then compared with predictions. While this report only includes comparisons for a subset of the configurations, the full database of all measurements and predictions is available in electronic format upon request. The results demonstrate that both conventional perforate-over-honeycomb and extended-reaction liners can be accurately modeled using COMSOL. Therefore, this modeling tool can be used with confidence to supplement the current suite of acoustic propagation codes, and ultimately develop new acoustic engine liners designed to reduce aircraft noise.
\end{abstract}

\section{Introduction}

A IRCRAFT noise can have a detrimental impact on public health in communities near airports. As a result, many airports limit operations (i.e., takeoffs and landings) to reduce community noise exposure. ${ }^{1}$ These restrictions inhibit the expansion of the aviation sector, which is an important driver of the U.S. economy. The development of new technologies capable of reducing aircraft noise could significantly reduce noise exposure and eliminate the need for these restrictions. One technology that is commonly used to reduce noise from the engines, and more specifically from ducted fans, is the acoustic engine liner. Conventional liners, which are typically installed in the engine nacelle, are resonant absorbers that are very effective for tonal noise. However, modern high-bypass-ratio engines also have a significant broadband component, and therefore new extended-reaction liners are being developed to attenuate broadband, as well as tonal noise. To facilitate the development of these new liners and ultimately reduce aircraft noise, manufacturers need access to flexible, accurate, and efficient modeling tools that are able to capture the effect of new liners on radiated noise.

Numerous research tools have been developed to model the effect of conventional acoustic liners on sound propagation through an engine nacelle. Most use a two-step approach in which the treatment is first represented as an acoustic impedance that is then applied as a boundary condition within the duct propagation code. Different propagation codes are used for different applications. Efficient codes that use a parabolic approximation to the convected Helmholtz equation, such as CDUCT-LaRC, are well-suited for large models and for optimization studies where computational efficiency is critical. ${ }^{2}$ Less restrictive codes that solve a finite element system using a direct solver strategy are also available, but tend to be more computationally demanding. ${ }^{3}$ While researchers have shown that the standard two-step approach can be used to accurately capture the effect of conventional liners on sound propagation in an engine nacelle, the approach is restricted to locally-reacting acoustic treatment that can be

${ }^{1}$ Research Engineer, Structural Acoustics Branch, Research Directorate, AIAA Member.

${ }^{2}$ Senior Research Scientist, Structural Acoustics Branch, Research Directorate, AIAA Associate Fellow.

${ }^{3}$ Acoustic Test Engineer, Boeing Test \& Evaluation, MS 1W-03, AIAA Member.

American Institute of Aeronautics and Astronautics 
represented as a surface impedance. In general, extended-reaction designs, which support lateral wave propagation inside the liner, cannot be modeled as a surface impedance. Instead, more general finite element codes are needed to explicitly model the wave propagation in the liner and flow duct in a single step. Commercially-available finite element packages could be used; however, many have not been rigorously validated for acoustic engine liner predictions. Rigorous evaluation is essential to gain trust in the predictions and establish the range of applicability of the code. Therefore, the goal of this study is twofold: to determine if one particular finite element package, COMSOL Multiphysics, can be used to accurately model engine liners including extended-reaction designs; and to provide a complete set of test data for different types of engine liners that can be used for future code evaluation activities.

This paper begins with a description of the liner samples tested. The experimental setup is then discussed and the test matrix is described. The finite element model is then presented. The accuracy of the modeling tool is assessed by comparing predictions with measurements, and finally conclusions are made concerning the applicability of the tool for acoustic engine liner simulations.

\section{Experimental Setup}

In order to assess the accuracy of modeling tools, such as COMSOL Multiphysics, an experimental database was compiled for several types of engine liners and operating conditions. Specifically, liner samples ranging from conventional locally-reacting liners to extended-reaction designs were installed in one wall of a flow duct where they were exposed to high sound pressure levels and grazing flow. The effect of the liner on the sound field in the flow duct was then measured using an array of microphones embedded in the walls of the duct. These measurements were then compared with predictions to determine the range of applicability of the modeling tool. The remainder of this section includes a description of each type of liner tested, as well as an overview of the test setup, and a description of the full test matrix.

\section{A. Test Liners}

In general, acoustic engine liners consist of three components, as shown in Fig. 1. Liners typically contain a perforated facesheet, which is the outermost portion of the liner exposed to flow. The facesheet is attached to the core, which is typically a honeycomb matrix. The honeycomb provides mechanical support for the facesheet while also preventing lateral wave propagation in the core (i.e., wave propagation parallel to the facesheet). Liners with honeycomb cores are typically referred to as locally-reacting liners, since the sound cannot enter the liner at one point and exit at another location. In contrast, if internal partitions are not included in the core then the liner is referred to as an extended-reaction liner. The final component of a typical liner is the backplate, which is assumed to be rigid.

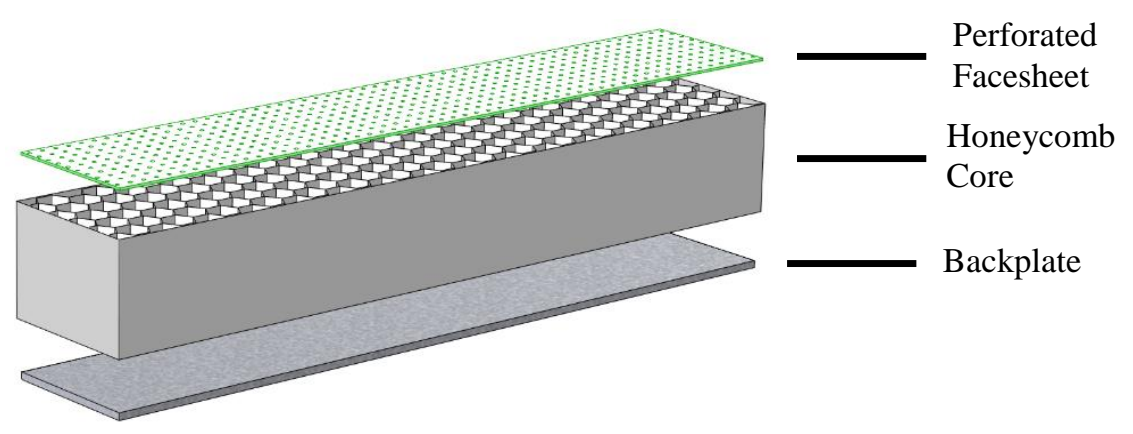

Figure 1. Diagram of a typical perforate-over-honeycomb core engine liner.

Nine liner configurations were tested as part of this evaluation. Note that all liners are uniform, with no significant variation along their width or length. Table 1 lists the facesheet, core, and overall length of each liner. The width is not included in the table, however, since all samples extend across the entire 2" width of the flow duct. The first sample is a relatively simple locally-reacting liner with a wire mesh over a conventional honeycomb core. The honeycomb is composed of hexagonal channels with a flat-to-flat dimension of 0.375 ". The wire mesh is used because it is well characterized with a known DC flow resistance of 270 MKS Rayls. The second sample contains a standard perforated facesheet with in-line 0.039 "-diameter circular holes over a honeycomb core. The facesheet is 0.032 " thick and has a porosity of 7.9\%. This type of liner is representative of the liners currently flown on aircraft. The third sample is slightly deeper (2" instead of 1.5"), but has no partitions in the core and is therefore, an extended-reaction liner. This sample is included to determine if the modeling tool is able to capture the lateral wave propagation in the core. The fourth sample is similar, but contains a different facesheet. In this case, the perforate is 0.032 " thick and has

American Institute of Aeronautics and Astronautics 
a porosity of $5 \%$. The facesheet has 0.012 "-diameter circular holes arranged in a staggered pattern (at an angle of 30 degrees with respect to the flow direction). This sample is included to assess the impact of different facesheet properties. The fifth sample has no facesheet and the core is filled with a bulk material composed of ceramic fibers. Liners containing bulk materials have historically been difficult to model using conventional modeling tools since the liner is not locally reacting. The sixth sample contains the $7.9 \%$ perforate over a 2 "-thick core filled with the same ceramic fibers. The seventh configuration is similar, but contains a 1" air gap in the core, between the perforate and bulk material. The eighth and ninth samples both use the 5\% perforate. The core of these samples contains an air gap adjacent to the perforate, along with a second type of bulk material composed of polymer fibers. In these samples, the bulk material is either 0.5 " or 1 " thick, as specified in Table 1 . These samples were included so that the benchmark dataset would contain two types of perforated facesheets and two types of bulk materials.

Table 1. Description of the liner samples.

\begin{tabular}{|c|c|c|c|c|c|c|}
\hline \multirow[b]{2}{*}{$\begin{array}{l}\text { Identifier } \\
\text { Prefix }\end{array}$} & \multicolumn{4}{|c|}{ Facesheet } & \multirow[b]{2}{*}{ Core } & \multirow[b]{2}{*}{$\begin{array}{c}\text { Active } \\
\text { Length } \\
\text { (in) }\end{array}$} \\
\hline & Type & $\begin{array}{c}\text { Porosity } \\
(\%)\end{array}$ & $\begin{array}{c}\text { Hole } \\
\text { Diameter, } \\
d \text { (in) }\end{array}$ & $\begin{array}{c}\text { Thickness, } \\
t \text { (in) }\end{array}$ & & \\
\hline C1R27 & $\begin{array}{c}\text { Mesh (270 } \\
\text { MKS Rayls) }\end{array}$ & $\mathrm{n} / \mathrm{a}$ & $\mathrm{n} / \mathrm{a}$ & $\mathrm{n} / \mathrm{a}$ & $\begin{array}{c}1.5 \% \\
\text { Honeycomb }\end{array}$ & 23.5 \\
\hline NBC2 & Perforate & 7.9 & 0.039 & 0.032 & $\begin{array}{c}1.5 " \\
\text { Honeycomb }\end{array}$ & 21.5 \\
\hline NBC1 & Perforate & 7.9 & 0.039 & 0.032 & 2" Air & 21.5 \\
\hline NSE & Perforate & 5 & 0.012 & 0.032 & 2" Air & 23.5 \\
\hline NBC5 & None & $\mathrm{n} / \mathrm{a}$ & $\mathrm{n} / \mathrm{a}$ & $\mathrm{n} / \mathrm{a}$ & $\begin{array}{l}\text { 2" Ceramic } \\
\text { Fiber }\end{array}$ & 21.5 \\
\hline NBC4 & Perforate & 7.9 & 0.039 & 0.032 & $\begin{array}{l}\text { 2" Ceramic } \\
\text { Fiber }\end{array}$ & 21.5 \\
\hline NBC3 & Perforate & 7.9 & 0.039 & 0.032 & $\begin{array}{c}\text { 1" Air / 1" } \\
\text { Ceramic Fiber }\end{array}$ & 21.5 \\
\hline NSN1 & Perforate & 5 & 0.012 & 0.032 & $\begin{array}{l}1.5 " \text { Air / } 0.5 " \\
\text { Polymer Fiber }\end{array}$ & 23.5 \\
\hline NSN2 & Perforate & 5 & 0.012 & 0.032 & $\begin{array}{c}\text { 1" Air / 1" } \\
\text { Polymer Fiber }\end{array}$ & 23.5 \\
\hline
\end{tabular}

\section{B. Grazing Flow Impedance Tube}

All of the tests were performed in the NASA Langley Research Center Grazing Flow Impedance Tube (GFIT), which is a 2.5 "-tall by 2"-wide waveguide that is used to measure the acoustic properties of liner samples exposed to high sound pressure levels and grazing flow (i.e., flow parallel to the facesheet). The bottom and sides of the duct are rigid, while a portion of the top wall can be replaced with the sample liner. While GFIT can be set up to simulate aft or inlet conditions, only aft duct propagation was considered in this study. In other words, the sound propagation and flow directions are the same. Flow is achieved using compressed air introduced through a plenum upstream of the sample, as well as a vacuum blower connected to the downstream termination section. The facility is capable of flow speeds up to Mach 0.6 and sound pressure levels up to $160 \mathrm{~dB}$, which are achieved using an array of twelve, 120-W acoustic drivers located in the upstream section. For this study, tests were performed at discrete frequencies from 400 to $3000 \mathrm{~Hz}$ in $200 \mathrm{~Hz}$ increments. Sound pressure measurements are collected using an array of flush-mounted microphones embedded in the walls of the duct. The array contains 91 microphones within the 40 "-long measurement section of the flow duct, as depicted in Fig. 2. The microphone data is processed using a cross-spectrum signal

American Institute of Aeronautics and Astronautics 
extraction method to determine the magnitude and phase (relative to a common upstream reference microphone) at each microphone location. Aft of the measurement section, the flow duct is terminated by a quasi-anechoic diffuser.

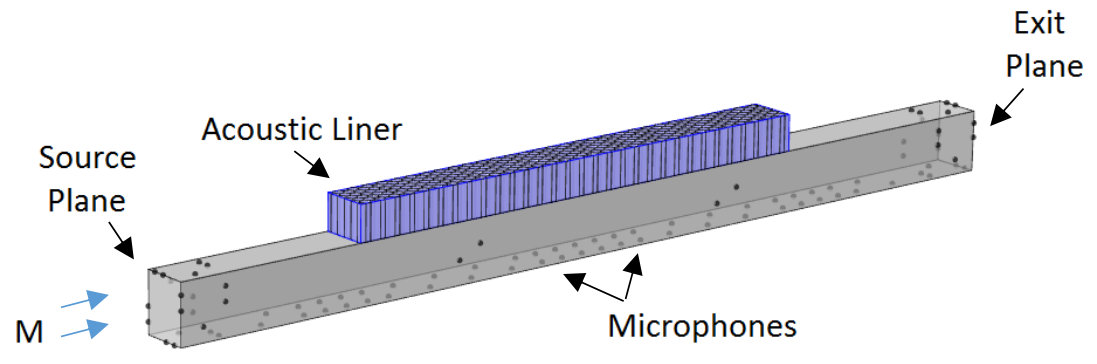

Figure 2. Sketch of the GFIT measurement section.

\section{Test Matrix}

In an effort to identify the range of applicability of the modeling tool, multiple types of liners were evaluated at different source sound pressure levels and grazing flow velocities. The sound pressure level and grazing flow velocity were varied to capture nonlinearities associated with the level and flow speed, respectively. Set-point centerline Mach numbers $\left(M_{\mathrm{C} / \mathrm{L}}\right)$ of $0,0.3$, and 0.5 were used, which correspond to cross-section-averaged Mach numbers of 0, 0.257, and 0.395 , respectively. Note that while the term "average Mach number" is often referred to as "bulk Mach number" in the literature, the first term is preferred in this report to make it clear that this parameter does not refer to the bulk material. While the full test matrix, shown in Table 2, includes 36 different configurations, this report only compares measurements and predictions for a representative subset of those cases, which are indicated with bold font. The full database is available, however, in electronic format upon request. This database contains all sound pressure level (SPL) and phase measurements collected in the flow duct, as well as the corresponding numerical predictions. 
Table 2. Test matrix (results from the tests in bold are discussed in detail in this report).

\begin{tabular}{|c|c|c|c|c|c|c|}
\hline Test & Identifier & Facesheet & Core & $\begin{array}{c}\text { Active } \\
\text { Length } \\
\text { (in) }\end{array}$ & $\begin{array}{c}\text { Set- } \\
\text { Point } \\
M_{\mathrm{C} / \mathrm{L}} \\
\end{array}$ & $\begin{array}{c}\text { Source } \\
\text { SPL } \\
\text { (dB) } \\
\end{array}$ \\
\hline 1 & C1R27M000L2 & Mesh & 1.5" Honeycomb & 23.5 & $\mathbf{0}$ & 120 \\
\hline 2 & C1R27M000L4 & Mesh & 1.5" Honeycomb & 23.5 & 0 & 140 \\
\hline 3 & C1R27M300L2 & Mesh & 1.5” Honeycomb & 23.5 & 0.3 & 120 \\
\hline 4 & C1R27M300L4 & Mesh & 1.5" Honeycomb & 23.5 & 0.3 & 140 \\
\hline 5 & NBC2M000L2 & Perforate (7.9\%) & 1.5" Honeycomb & 21.5 & $\mathbf{0}$ & 120 \\
\hline 6 & NBC2M000L4 & Perforate $(7.9 \%)$ & 1.5" Honeycomb & 21.5 & 0 & 140 \\
\hline 7 & NBC2M300L2 & Perforate (7.9\%) & 1.5" Honeycomb & 21.5 & 0.3 & 120 \\
\hline 8 & NBC2M300L4 & Perforate $(7.9 \%)$ & 1.5" Honeycomb & 21.5 & 0.3 & 140 \\
\hline 9 & NBC2M500L2 & Perforate $(7.9 \%)$ & 1.5" Honeycomb & 21.5 & 0.5 & 120 \\
\hline 10 & NBC2M500L4 & Perforate $(7.9 \%)$ & 1.5" Honeycomb & 21.5 & 0.5 & 140 \\
\hline 11 & NBC1M000L2 & Perforate $(7.9 \%)$ & 2" Empty & 21.5 & 0 & 120 \\
\hline 12 & NBC1M000L4 & Perforate $(7.9 \%)$ & 2" Empty & 21.5 & 0 & 140 \\
\hline 13 & NBC1M300L2 & Perforate $(7.9 \%)$ & 2" Empty & 21.5 & 0.3 & 120 \\
\hline 14 & NBC1M300L4 & Perforate $(7.9 \%)$ & 2" Empty & 21.5 & 0.3 & 140 \\
\hline 15 & NBC1M500L2 & Perforate $(7.9 \%)$ & 2" Empty & 21.5 & 0.5 & 120 \\
\hline 16 & NBC1M500L4 & Perforate $(7.9 \%)$ & 2" Empty & 21.5 & 0.5 & 140 \\
\hline 17 & NSEM000L3 & Perforate $(5 \%)$ & 2" Empty & 23.5 & 0 & 130 \\
\hline 18 & NSEM300L3 & Perforate $(5 \%)$ & 2" Empty & 23.5 & 0.3 & 130 \\
\hline 19 & NBC5M000L2 & None & 2" Ceramic Fiber & 21.5 & $\mathbf{0}$ & 120 \\
\hline 20 & NBC5M000L4 & None & 2" Ceramic Fiber & 21.5 & 0 & 140 \\
\hline 21 & NBC4M000L2 & Perforate $(7.9 \%)$ & 2" Ceramic Fiber & 21.5 & 0 & 120 \\
\hline 22 & NBC4M000L4 & Perforate $(7.9 \%)$ & 2" Ceramic Fiber & 21.5 & 0 & 140 \\
\hline 23 & NBC4M300L2 & Perforate (7.9\%) & 2" Ceramic Fiber & 21.5 & 0.3 & 120 \\
\hline 24 & NBC4M300L4 & Perforate $(7.9 \%)$ & 2" Ceramic Fiber & 21.5 & 0.3 & 140 \\
\hline 25 & NBC4M500L2 & Perforate $(7.9 \%)$ & 2" Ceramic Fiber & 21.5 & 0.5 & 120 \\
\hline 26 & NBC4M500L4 & Perforate $(7.9 \%)$ & 2" Ceramic Fiber & 21.5 & 0.5 & 140 \\
\hline 27 & NBC3M000L2 & Perforate $(7.9 \%)$ & 1" Air / 1" Ceramic Fiber & 21.5 & 0 & 120 \\
\hline 28 & NBC3M000L4 & Perforate $(7.9 \%)$ & 1" Air / 1" Ceramic Fiber & 21.5 & 0 & 140 \\
\hline 29 & NBC3M300L2 & Perforate $(\mathbf{7 . 9 \%})$ & 1" Air / 1" Ceramic Fiber & 21.5 & 0.3 & 120 \\
\hline 30 & NBC3M300L4 & Perforate $(7.9 \%)$ & 1" Air / 1" Ceramic Fiber & 21.5 & 0.3 & 140 \\
\hline 31 & NBC3M500L2 & Perforate $(7.9 \%)$ & 1" Air / 1" Ceramic Fiber & 21.5 & 0.5 & 120 \\
\hline 32 & NBC3M500L4 & Perforate $(7.9 \%)$ & 1" Air / 1" Ceramic Fiber & 21.5 & 0.5 & 140 \\
\hline 33 & NSN1M000L3 & Perforate $(5 \%)$ & $1.5 "$ Air / 0.5" Polymer Fiber & 23.5 & 0 & 130 \\
\hline 34 & NSN1M300L3 & Perforate $(5 \%)$ & $1.5 "$ Air / 0.5" Polymer Fiber & 23.5 & 0.3 & 130 \\
\hline 35 & NSN2M000L3 & Perforate $(5 \%)$ & 1" Air / 1" Polymer Fiber & 23.5 & 0 & 130 \\
\hline 36 & NSN2M300L3 & Perforate $(5 \%)$ & 1" Air / 1" Polymer Fiber & 23.5 & 0.3 & 130 \\
\hline
\end{tabular}




\section{Model Setup}

The predictions presented in this report were generated using the Acoustics Module within COMSOL Multiphysics 5.2. Since individual models were created for each test listed in Table 2, COMSOL's scripting capability, LiveLink for MATLAB, was used to parametrically generate each model, solve the system of equations, and then post-process the results. The modeling procedure itself was relatively standard. The geometry was first defined, then the appropriate physics (i.e., differential equations) were selected, the boundary conditions were defined, the domain was meshed, and finally the equations were solved. Each of these steps are discussed in more detail below.

\section{A. Geometry}

Since the goal of this study is to assess the accuracy of acoustic liner models generated with COMSOL, the models were created to mimic the test setup. Specifically, three-dimensional models were created that capture the relevant geometric features of the 40 "-long measurement section. The coordinate system used in the model matches the standard GFIT coordinate system where $\mathrm{x}$ is axial, $\mathrm{y}$ is vertical, and $\mathrm{z}$ is spanwise across the duct, as shown in Fig. 3. Once again, the model (and test) simulate an aft-duct configuration in which the sound propagates downstream. Therefore, the flow direction corresponds to the positive $\mathrm{x}$-direction. The origin is shown by the black dot in the lower left corner of Fig. 3. The source plane corresponds to $x=0$ " and the termination plane corresponds to $x=40$ ". In all cases, the leading edge of the liner is 8.25 " downstream of the source plane to be consistent with the tests.

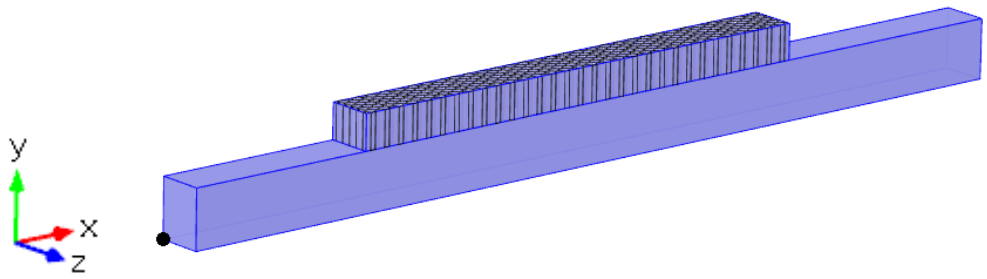

Figure 3. Geometric model of the duct and perforate-over-honeycomb core liner.

\section{B. Physics}

A two-step, uncoupled approach is used to solve the model. The first step is to solve for the mean flow field and the second step is to solve for the acoustic perturbations on top of the mean flow. The mean flow field is determined using compressible potential flow equations, which assume that the fluid is compressible and inviscid. The governing equations are then linearized around the background mean flow and perturbation theory is used to derive linearized potential flow equations for the acoustic perturbations. Since the present implementation of the linearized potential flow equations does not support complex-valued fluid properties, an additional set of pressure acoustic equations are also included in order to model the bulk materials. The following subsections describe the modeling approach in more detail, starting with the compressible potential flow formulation. Note that while this report only contains an overview of the equations solved, additional details are available in the COMSOL Acoustics Module Users Guide. ${ }^{4}$

\section{Compressible Potential Flow}

The first step in the analysis is to solve for the mean flow variables (i.e., density and velocity potential). For the configurations considered in this study, the solution is trivial. The flow duct has a constant cross-section (with no flow entrained in the liner) and the flow is assumed to be uniform at both the source and termination planes. Therefore the background flow field will be uniform throughout the flow duct, and the density, speed of sound, and background velocity could be calculated directly from the temperature and static pressure measurements acquired during the test. However, since the compressible potential flow equations are also applicable for more complex configurations with nonuniform flow fields (as long as the flow is irrotational), the full compressible flow equations are used in this study.

Governing Equations - The derivation of the compressible potential flow equations starts with the equations governing the conservation of mass and momentum for an inviscid fluid. An equation of state for an ideal gas (assuming the flow is isentropic) is then included to close the system of equations. If the flow field is assumed to be irrotational, then the velocity potential field, $\Phi$, can be defined such that $\boldsymbol{V}=\nabla \Phi$, where $\boldsymbol{V}$ is the mean velocity field. The stationary form of the compressible potential flow equations can then be written as 


$$
\left(\frac{|\nabla \Phi|^{2}}{2}+\frac{\gamma}{\gamma-1} \frac{\rho^{\gamma-1} P_{0}}{\rho_{0}^{\gamma}}\right)=\frac{V_{0}^{2}}{2}+\frac{\gamma P_{0}}{(\gamma-1) \rho_{0}}
$$

and

$$
\nabla \cdot(\rho \nabla \Phi)=0
$$

where $\nabla$ denotes the gradient, $\Phi$ is the velocity potential field, $\gamma$ is the specific heat ratio, $\rho$ is the mean background density, $\rho_{0}$ is the reference density, $P_{0}$ is the reference pressure, and $V_{0}$ is the reference velocity. Note that this set of simplified equations assumes that negligible gravity forces act on the fluid, which is an appropriate assumption for the horizontal flow duct. For this analysis, the static state of the fluid is used as the reference state. Since the goal of the study is to evaluate the modeling tool, the models are setup to match the conditions present during the test. Specifically, the reference pressure was specified to match the static pressure $P_{s}$ measured at the midpoint of the liner during the test. The reference speed of sound was defined as $c_{0}=\sqrt{\gamma R T_{s}}$, where $\gamma=1.4, R=287 \mathrm{~J} /(\mathrm{kg} \mathrm{K})$, and $T_{s}$ is the static temperature in kelvins. Similarly, the reference density was defined as $\rho_{0}=P_{s} /\left(R T_{s}\right)$, and the reference velocity was specified as $M c_{0}$, where $M$ is the average Mach number in the flow duct.

Boundary Conditions - The mean flow field is the solution to the compressible potential flow equations satisfying the appropriate boundary conditions at the source plane, duct walls, and termination plane. The boundary condition at the source plane is defined in terms of the mass flow per unit area, which is assumed to be uniform over the plane and is defined as the product of the normal velocity and density, $M c_{0} \rho_{0}$. The flow exits the domain through the termination plane, which is also constrained to have normal flow (i.e., zero tangential velocity). The normal component of velocity at all other boundaries was set to zero.

\section{Linearized Potential Flow}

The second step in the analysis is to solve for the acoustic perturbations on top of the mean flow. This is accomplished by solving linearized potential flow equations subject to the appropriate boundary conditions.

Governing Equations - The conservation of mass and momentum are used along with the equation of state for an ideal gas (assuming the flow is isentropic). The equations are then decomposed into time-independent and acoustic components, and linearized about the mean flow (neglecting nonlinear terms). In this case, the acoustic potential, $\phi$, is related to the particle velocity by $\mathbf{v}=\nabla \phi$. The second order wave equation for nonuniform mean flow can then be written as

$$
-\frac{\rho}{c_{0}^{2}} i \omega(i \omega \phi+\boldsymbol{V} \cdot \nabla \phi)+\nabla \cdot\left[\rho \nabla \phi-\frac{\rho}{c_{0}^{2}}(i \omega \phi+\boldsymbol{V} \cdot \nabla \phi) \boldsymbol{V}\right]=0
$$

which is commonly referred to as the convected Helmholtz equation (expressed here in the frequency domain). ${ }^{3}$ The equation can be solved, subject to the boundary conditions, to obtain the acoustic potential. Once the acoustic potential is known, the acoustic pressure can be found as

$$
p=-\rho(i \omega \phi+\boldsymbol{V} \cdot \nabla \phi)
$$

Boundary Conditions - Once again, since the goal of this study is to assess the accuracy of a commercial finite element package, it is useful to match the acoustic boundary conditions used in the model to the test. This is accomplished by prescribing the magnitude and phase of the total pressure at both the source and termination planes (in terms of the acoustic potential) to match the measured values at similar locations. While the termination plane impedance could be set to $\rho c$ to avoid the need for downstream measured data, this would bias the predictions since the actual termination of the flow duct is not truly anechoic (as demonstrated by the standing wave pattern in the pressure measurements downstream of the liner). To eliminate this bias, the acoustic pressure at the termination plane is defined in the model to match the acoustic pressure measured during the test. While multiple microphones are located at both the source and exit planes, as shown in Fig. 2, the spatial variation was not input into the model. Instead, a single measurement at each end of the flow duct was used to define a uniform sound field at the source and exit planes. Note that this approximation is only valid below the frequency where the first higher-order mode cuts on in the flow duct.

As mentioned previously, the model is defined in terms of the linearized potential flow equations. Therefore, the boundary conditions must be expressed in terms of the acoustic potential. When the background velocity is zero, the acoustic potential (Eq. (4)) can be uniquely determined for a specified acoustic pressure. However, when the background velocity is non-zero, the acoustic pressure depends on both the acoustic potential (prescribed) and its

American Institute of Aeronautics and Astronautics 
gradient, which is computed. Therefore, in general, the acoustic potential corresponding to a given total acoustic pressure cannot be found a priori. This issue is addressed by iteratively updating the applied acoustic potential (at the source and termination planes) until the computed pressure corresponds to the desired value. This can be automated in the software by using a global equation node.

In addition to the boundary conditions on each end of the flow duct, acoustically rigid walls are defined along the perimeter of the flow duct and on the sides and back of the liner. A similar interior boundary condition is also included to represent different components of the liner. For instance, the interior partitions in the honeycomb core are assumed to be rigid, with an infinite impedance. This implies that the pressure on either side of the partition can be different.

The perforated facesheet is modeled in a similar way; however, instead of assuming an infinite impedance, the facesheet impedance is defined as a transfer impedance. To be clear, the perforated facesheet is not explicitly modeled within the finite element model, but is instead represented as an interior impedance. The impedance corresponds to the ratio of the pressure drop across the facesheet (i.e., $p_{1}-p_{2}$ ) relative to the normal component of the particle velocity at the surface of the facesheet. For this study, the transfer impedances for the perforated facesheets were estimated using the two-parameter model, which is discussed in detail by Ref. 5 and is summarized in Appendix A. Note that the transfer impedance of the facesheet depends on the acoustic velocity at the surface of the liner, which in turn is dependent on the facesheet impedance. Therefore, an iterative approach was used to simultaneously determine both the transfer impedance and acoustic velocity, as described by Ref. 5 .

\section{Pressure Acoustics}

Since the linearized potential flow equations that are implemented in COMSOL 5.2 do not support complex-valued fluid properties, the bulk material was represented using pressure acoustic equations as discussed below.

Governing Equations - The derivation of the pressure acoustic equations is similar to the previous section, however in this case, the fluid is assumed to be stationary. As a result, the frequency domain formulation of the governing equation reduces to the Helmholtz equation (with source terms removed)

$$
-\frac{1}{\rho_{c}} \nabla^{2} p-\frac{\omega^{2} p}{\rho_{c} c_{c}^{2}}=0
$$

where the bulk material is modeled using complex-valued density $\rho_{c}$ and speed of sound, $c_{c}$. The equivalent fluid values used in the models were experimentally determined using the two-thickness method, as described in Appendix B. For example, the equivalent fluid properties for the fibrous ceramic material are included in Fig. 4. Notice that both the effective density and speed of sound are frequency dependent and complex-valued. These parameters are provided in tabular form for both of the bulk materials considered in this study in Appendix C.
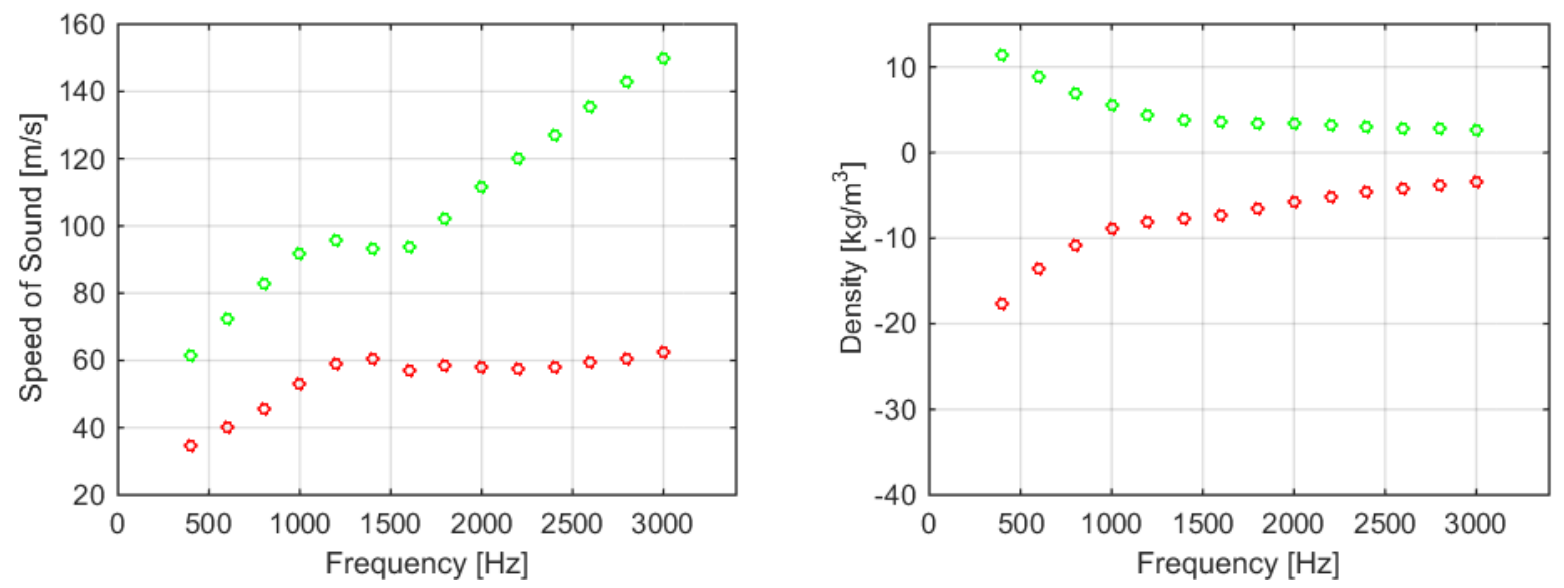

Figure 4. Complex speed of sound (left) and density (right) for the fibrous ceramic material. The green circles represent the real component while the red circles correspond to the imaginary component.

Boundary Conditions - Once again, boundary conditions are necessary to solve the governing equation. For the liners considered in this study, the bulk material was surrounded on the sides and back by rigid walls, which were modeled as acoustically rigid boundaries. In contrast, at the interface between the bulk material and the air within the

American Institute of Aeronautics and Astronautics 
liner, the pressure was assumed to be continuous. This was enforced by adding a symmetric pressure boundary condition at that interface.

\section{Mesh and Study Settings}

The size of the mesh varied based on the type of liner under consideration. For liners with a honeycomb core, the element size was selected to adequately resolve the smallest geometrical features of the honeycomb matrix. This resulted in a mesh, composed of free tetrahedral elements, that was much denser than would be needed to resolve the acoustic field; however, since solve times were generally short, there was no need to coarsen the mesh. For configurations without the honeycomb core, the domain was meshed with free tetrahedral elements with a maximum element size of approximately $0.01 \mathrm{~m}$. While the software allows the user to specify the element order, the default quadratic setting was used for this analysis. Therefore, each tetrahedral element was represented internally using 10 nodes.

All models contained three dependent variables per node (i.e., density, mean flow velocity potential, and either acoustic potential or acoustic pressure), however two of the variables (density and mean flow velocity potential) were solved upfront during the first step of the analysis. This step, which consisted of solving the compressible potential flow equations for the mean flow variables, was relatively quick, taking less than 30 seconds on a standard laptop (Intel Core i7-2720QM CPU @ 2.20GHz with 16 GB of RAM). Therefore, it was only necessary to solve for one variable (either acoustic potential or pressure) during the second step of the analysis. Specifically, the acoustic response was found using a direct frequency domain solution from $400 \mathrm{~Hz}$ to $3000 \mathrm{~Hz}$ in $200 \mathrm{~Hz}$ increments. The solution time for this step was approximately 10 seconds per frequency using a standard laptop. However, recall that an iterative process had to be used to determine the transfer impedance for the perforated facesheet. Since no more than 3 iterations were typically required to obtain a good estimate of the transfer impedance, the average solve time for the full frequency domain solution was approximately 400 seconds.

\section{Results and Discussion}

Recall that the primary goal of this paper is to evaluate the accuracy of acoustic liner models built using a commercially-available finite element package. However, since the acoustic boundary conditions used in the model (at the source and termination planes) are specified based on test data, the figure of merit cannot simply be liner attenuation, or the difference between the sound pressure level (SPL) at the source and termination planes. Instead, the models are assessed by qualitatively comparing measurements and predictions of the sound pressure level as a function of position along the duct. In addition to this qualitative comparison, a prediction quality metric is also used to quantify the fit. This section begins with a description of the prediction quality metric. The results for five representative liner configurations are then discussed.

\section{A. Prediction Quality Metric}

The agreement between predictions and measurements is quantified using a prediction quality metric, which is defined as

$$
P Q M=-20 \log _{10}\left(\frac{1}{\left\|p\left(x_{1}\right)_{\text {meas }}\right\|}\left(\frac{1}{N} \sum_{I=1}^{N}\left\|p\left(x_{I}\right)_{\text {num }}-p\left(x_{I}\right)_{\text {meas }}\right\|\right)\right)
$$

where $p$ is the complex pressure, $N$ is the number of microphones in the measurement section, $x_{I}$ is the axial location of the $I^{\text {th }}$ microphone, such that $x_{1}$ corresponds to the axial location of the reference microphone in the source plane, meas denotes measurement, num denotes numerical predictions, and $\|$ || corresponds to the complex absolute value. Note that large values of the prediction quality metric signify good agreement, while small values indicate that there are significant discrepancies between measurements and predictions. As an example, the prediction quality metric is plotted in Fig. 5 as a function of frequency for one configuration (i.e., Test 1: wire mesh over honeycomb core). As the figure shows, the fit is very good at most frequencies, exceeding $20 \mathrm{~dB}$. In other words, at most frequencies the average of the absolute value of the difference between the prediction and measurement is less than $10 \%$ of the source plane amplitude (i.e., $\left.\left\|p\left(x_{1}\right)_{\text {meas }}\right\|\right)$.

American Institute of Aeronautics and Astronautics 


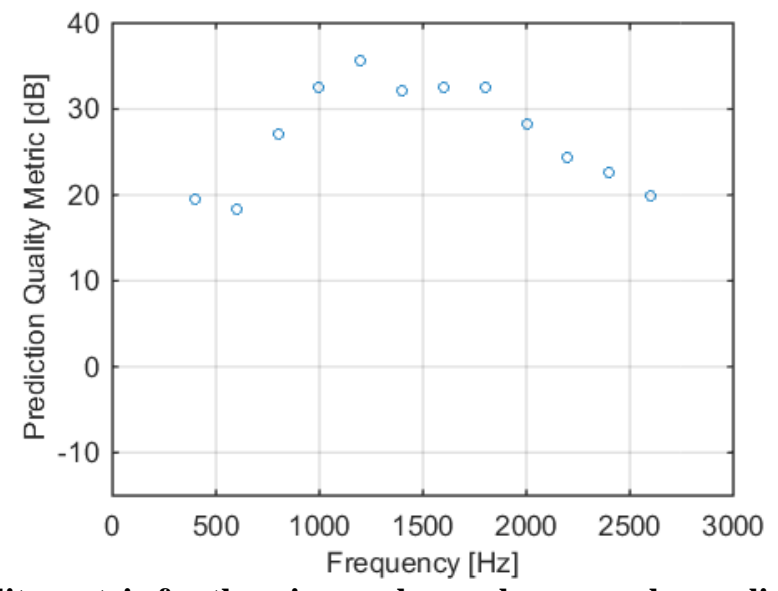

Figure 5. Prediction quality metric for the wire mesh over honeycomb core liner (Test 1, C1R27M000L2).

The comparison is distilled further into a single number for each configuration, which represents the median over all frequency bands, as shown in Fig. 6. The median is used, instead of the mean, to deemphasize the impact of extreme values at individual frequencies. This is important because predictions can be particularly sensitive to the accuracy of input parameters, such as facesheet resistance, near anti-resonances. At those frequencies, relatively small errors in the input parameters can result in large changes in the predictions. In addition to showing the median prediction quality versus test number, Fig. 6 also includes a horizontal line at $10 \mathrm{~dB}$ that represents the minimum acceptable prediction quality. Physically, the horizontal line represents the point where the median normalized error is approximately 0.3. The two sets of bars, shown in Fig. 6, will be discussed in detail in subsequent sections, but for now it is useful to note that the bars in yellow correspond to the baseline predictions, while the bars in green show the median prediction quality for models with updated input parameters (i.e., updated empirical parameters for the $7.9 \%$ porosity facesheet).

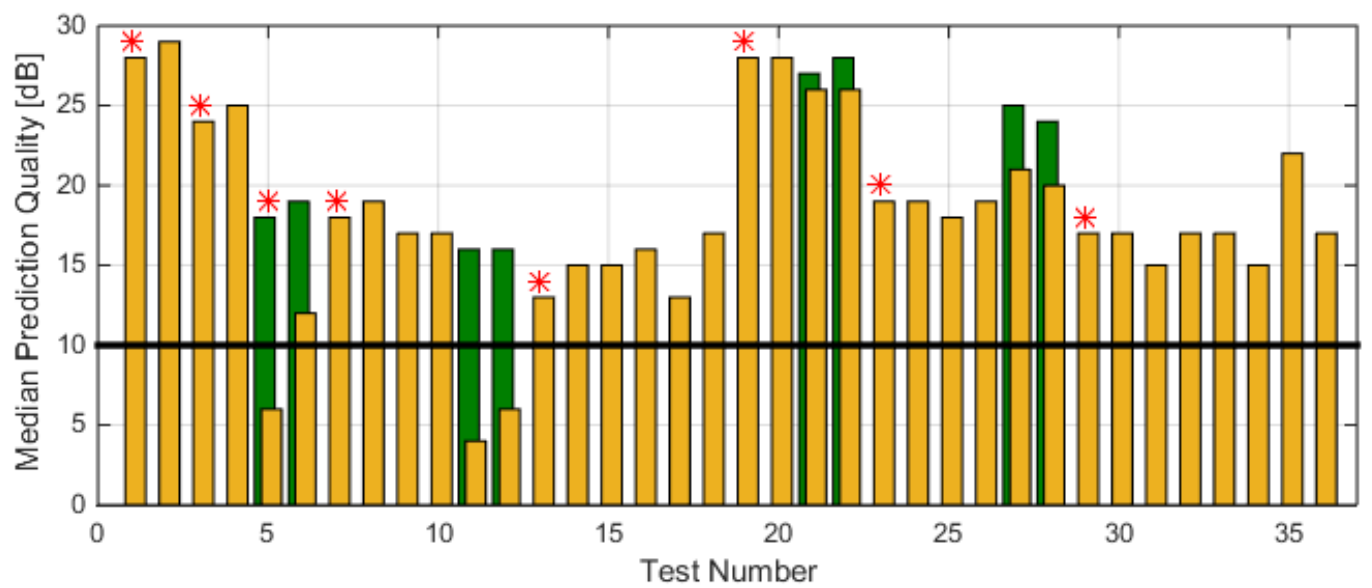

Figure 6. Median prediction quality for each test. The bars in yellow were calculated using default input parameters, while the bars in green were generated using updated input parameters for the $7.9 \%$ porosity facesheet. The red asterisks denote the tests that are discussed in detail in this report.

Recall that all of the test cases are not discussed in detail in this report. Instead, a representative sample of the tests are discussed to highlight important trends. The first configuration discussed is the wire-mesh-over-honeycomb core liner, labeled as Test 1.

\section{B. Wire Mesh over Honeycomb (Tests 1 and 3)}

The first liner configuration had a wire mesh facesheet over a standard honeycomb core. This relatively simple, locally-reacting liner is particularly easy to model since the transfer impedance of the wire mesh can be approximated as a constant resistance (270 MKS Rayls in this case). In addition to having a constant impedance versus frequency, the wire mesh is also assumed to behave linearly, which means that the impedance is unaffected by the amplitude of the sound pressure level and the grazing flow Mach number.

American Institute of Aeronautics and Astronautics 
The first case considered, labeled as Test 1 , had a source level of $120 \mathrm{~dB}$ with no grazing flow. The accuracy of the model was initially assessed by comparing measurements and predictions of the sound pressure level as a function of position along the duct, as shown in Fig. 7. Multiple curves, corresponding to different frequencies, are included in the plots. In all cases, the markers indicate GFIT measurements while the solid lines correspond to the predictions. At both the source and termination planes, the pressure is defined in the model to match the measurements. Therefore, there should be no discrepancies between predictions and measurements at either end of the duct (i.e., $\mathrm{x}=0$ " and $\mathrm{x}=40$ "). Instead, the assessment is performed by comparing the shape of the curves between those two points. As the figure shows, the model accurately captures the relevant trends observed during Test 1 , both in terms of the slope of the attenuation and the standing wave patterns observed in the measurements. Before proceeding, it is useful to note that the y-axis, SPL, is plotted on a scale of 50 to $140 \mathrm{~dB}$ in all plots included in this report. While the large range has the undesired effect of obscuring small differences between measurements and predictions, it was selected so that the same scale could be used for all tests. The use of a common scale simplifies the comparison between tests, and was therefore used despite the previously mentioned disadvantage.
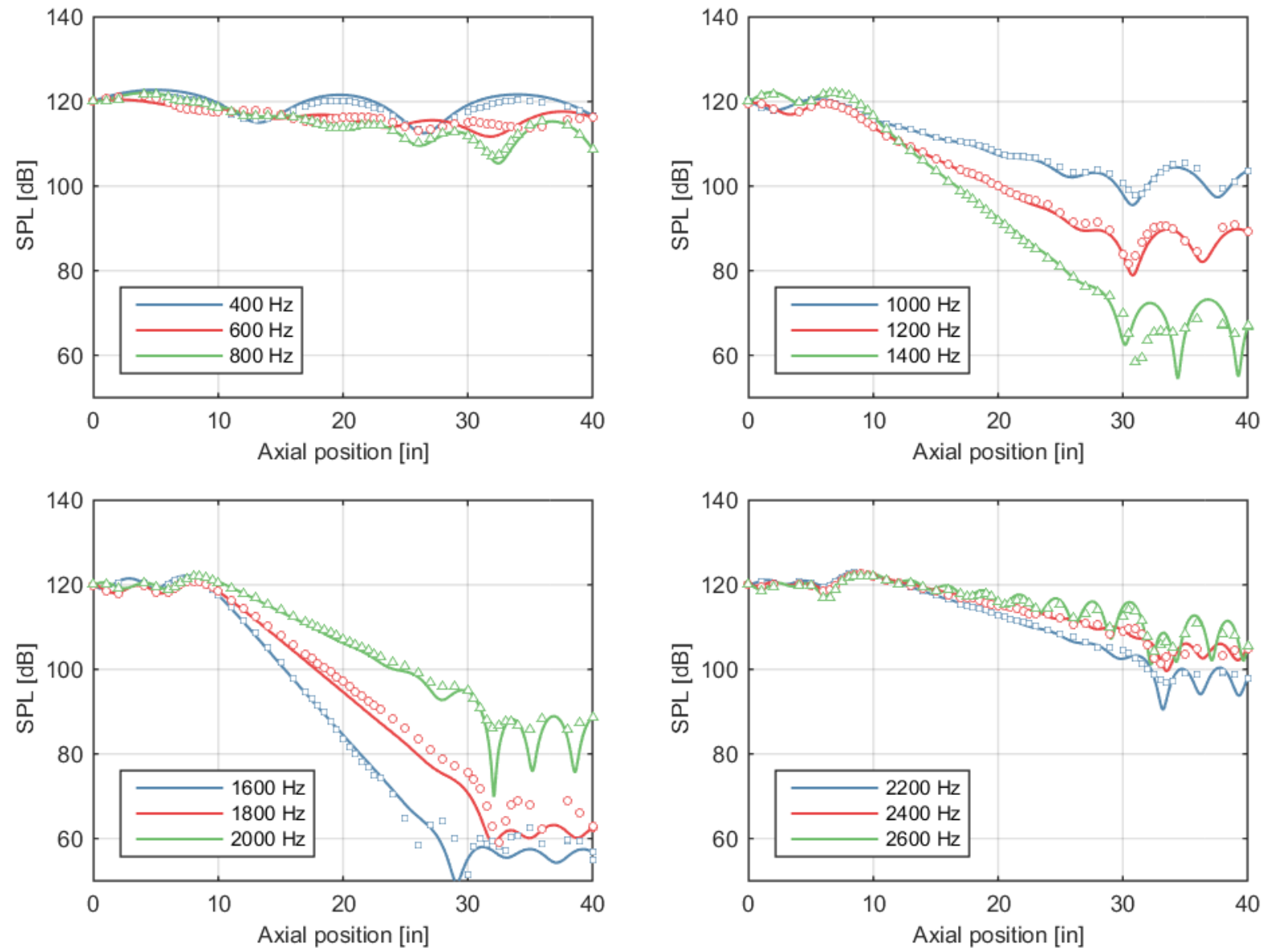

Figure 7. Sound pressure level versus position in the flow duct with the wire-mesh-over-honeycomb core liner (Test 1, C1R27M000L2). GFIT measurements are indicated with markers while the solid lines correspond to predictions.

Note that although tests (and predictions) were performed up to $3000 \mathrm{~Hz}$, the effect of higher-order modes is not captured by the model since plane wave boundary conditions are defined at the source and termination planes. Therefore, the comparison is only presented up to the frequency where the first higher-order mode cuts on. For the no-flow case, the first higher-order mode cuts on around $2710 \mathrm{~Hz}$, and therefore, results are only shown up to $2600 \mathrm{~Hz}$. Similarly, when $M_{\mathrm{C} / \mathrm{L}}=0.3$ the upper frequency for the comparison is $2400 \mathrm{~Hz}$ (since the first higher order mode cutson around $2590 \mathrm{~Hz}$ ), and when $M_{\mathrm{C} / \mathrm{L}}=0.5$ the upper limit is $2200 \mathrm{~Hz}$ (since the first higher order mode cuts-on around $2350 \mathrm{~Hz})$.

American Institute of Aeronautics and Astronautics 
To quantify the fit, the prediction quality metric was calculated using Eq. (6). However, as the equation shows, this metric is based on differences in the complex-valued pressure, and is therefore dependent on both the magnitude and phase of the response. Therefore, it is instructive to also consider Fig. 8, which shows the phase versus position in the flow duct. Recall that the pressure was defined in the model to match the measurements at both the source and termination planes. Therefore, there should be no discrepancies in phase between predictions and measurements at either end of duct. So once again, the comparison is performed by comparing the shape of the curves between those two points. It is clear from Fig. 8 that the phase predictions, like the magnitude predictions, capture the trends observed in the measurements. Therefore, it should not be surprising that the prediction quality metric, shown in Fig. 5, is relatively large, exceeding $20 \mathrm{~dB}$ at most frequencies. The median of the prediction quality metric for this case is $28 \mathrm{~dB}$, as shown in Fig. 6. Note that while additional phase plots are not included in the report, all of the magnitude and phase data is readily available upon request.
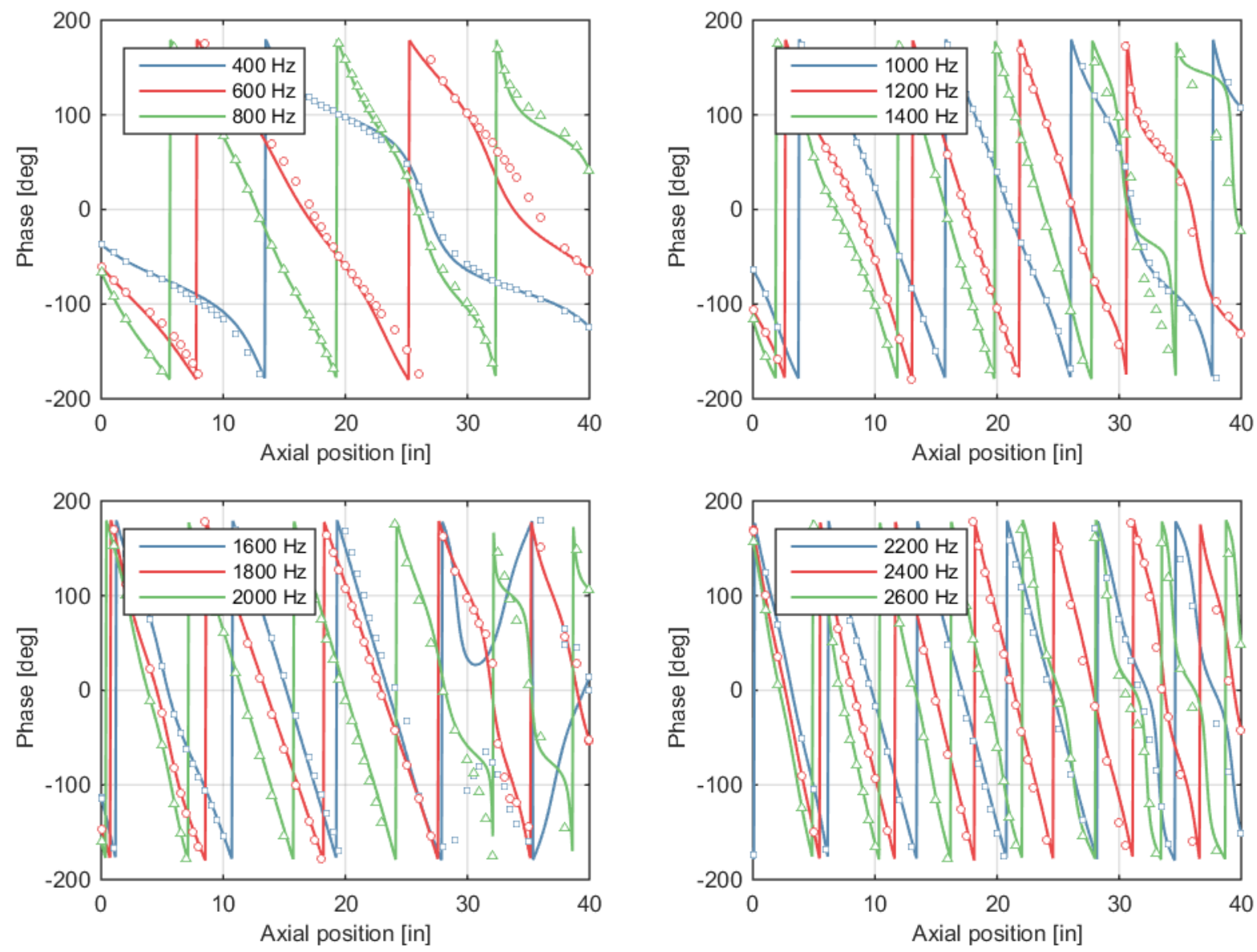

Figure 8. Phase versus position in the flow duct with the wire-mesh-over-honeycomb core liner (Test 1, C1R27M000L2). GFIT measurements are indicated with markers while the solid lines correspond to predictions.

The same liner was considered again in Test 3, but with grazing flow $\left(M_{\mathrm{C} / \mathrm{L}}=0.3\right)$. Since the liner includes a wire mesh facesheet that is assumed to be linear, the grazing flow Mach number was not expected to have any impact on the impedance of the liner. Instead, the flow was anticipated to only have an effect on the wave propagation in the flow duct, which is captured by the model. Therefore, the level of agreement between predictions and measurements was expected to be similar to Test 1 . The comparison of SPL versus position in the flow duct is shown in Fig. 9. Once again, the model captures the relevant trends observed in the test, although the median agreement of $24 \mathrm{~dB}$, shown in Fig. 6, is slightly worse (i.e., lower) than the no-flow case (Test 1). Recall that the model assumes that the flow is inviscid, and therefore the mean flow profile is assumed to be uniform across the duct. However, in reality, the velocity profile in the flow duct is not uniform, as discussed by Ref. 6 . Therefore, neglecting the shear-flow effects may have

American Institute of Aeronautics and Astronautics 
contributed to the observed discrepancies between measurements and predictions with flow. It is also possible that the wire mesh itself may not be perfectly linear. In other words, the actual impedance of the liner may be weakly dependent on the flow, which is an effect that is not captured by the current model.
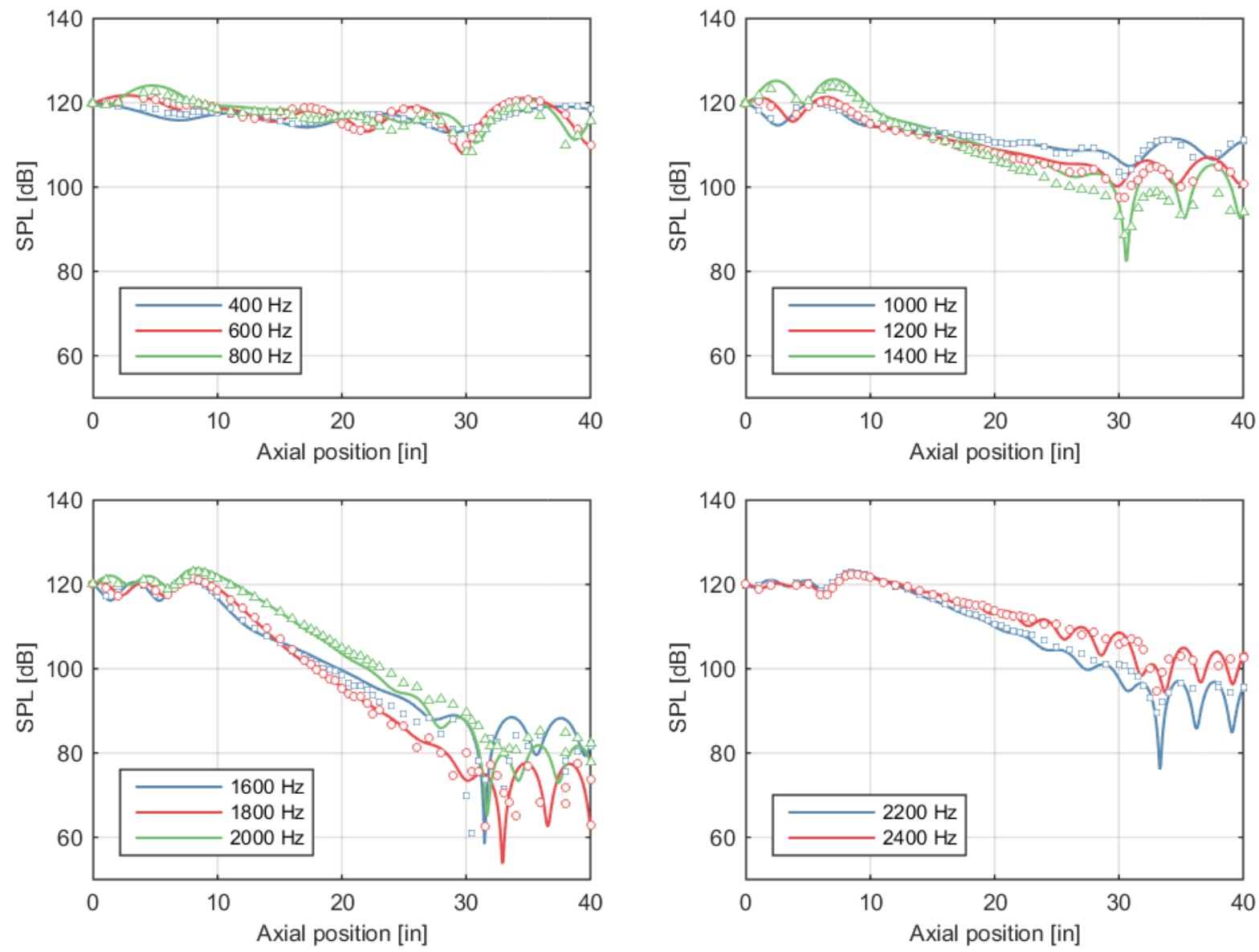

Figure 9. Sound pressure level versus position for the wire-mesh-over-honeycomb core liner with grazing flow at $M_{\mathrm{C} / \mathrm{L}}=0.3$ (Test 3, C1R27M300L2). GFIT measurements are indicated with markers while the solid lines correspond to predictions.

Before proceeding to the next liner configuration, it is useful to summarize the wire-mesh-over-honeycomb results. In general, the comparison between predictions and measurements is very good. There are some discrepancies, particularly in regions where the attenuation is very high; however, the overall trends observed in the measurements are captured by the predictions. By comparing the median prediction quality for Tests 1 and 2 in Fig. 6, it is clear that the level of agreement between predictions and measurements is largely independent of source level. The small differences in prediction quality may be attributed to test to test variability or may indicate that the liner impedance is weakly nonlinear with respect to source level. When grazing flow is included, the fit between predictions and measurements is reduced relative to the no-flow case. This could be attributed to the presence of shear flow in the flow duct, which is not captured in the model, or it could indicate that the wire mesh is weakly nonlinear with respect to the grazing-flow Mach number, which is also not captured in the present model.

\section{Perforate over Honeycomb (Tests 5 and 7)}

The next configuration considered is typical of a conventional liner. Specifically, the liner has a standard perforated facesheet with circular holes over a honeycomb core. While the transfer impedance of the wire mesh is linear, the impedance of perforated facesheets can be strongly affected by both the source SPL and the grazing-flow Mach number (e.g., see Ref. 6). Therefore, the interior (or transfer) impedance for the facesheet was estimated using a twoparameter model, summarized in Appendix A, which is a function of both SPL and grazing flow.

American Institute of Aeronautics and Astronautics 
Test 5 is considered next, which had a source level of $120 \mathrm{~dB}$ and no grazing flow. The initial comparison between predictions and measurements is shown in Fig. 10. Clearly, the model does not capture the effect of the liner as accurately as previous comparisons. Specifically, the predictions show a much more pronounced standing wave pattern than is seen in the measured data. In addition, the model significantly underpredicts the measured attenuation at $1400 \mathrm{~Hz}$. While the response at frequencies near maximum attenuation is often difficult to predict since small errors in the liner impedance are magnified, the discrepancy observed in this case is worth noting. For reference, the median prediction quality for Test 5 is only $6 \mathrm{~dB}$ (as shown in Fig. 6), which is more than $20 \mathrm{~dB}$ less than the configuration containing the wire mesh facesheet (Test 1 ). Since the model accurately predicted the sound field in the flow duct for Test 1, but not 5, the transfer impedance of the perforated facesheet was reassessed. Recall that the transfer impedance was predicted using a semi-empirical two-parameter model. This model was developed by industry to represent the impedance of typical liners in representative conditions. The no-flow condition is not a representative condition, and therefore it is logical to suspect inaccuracies in this case.
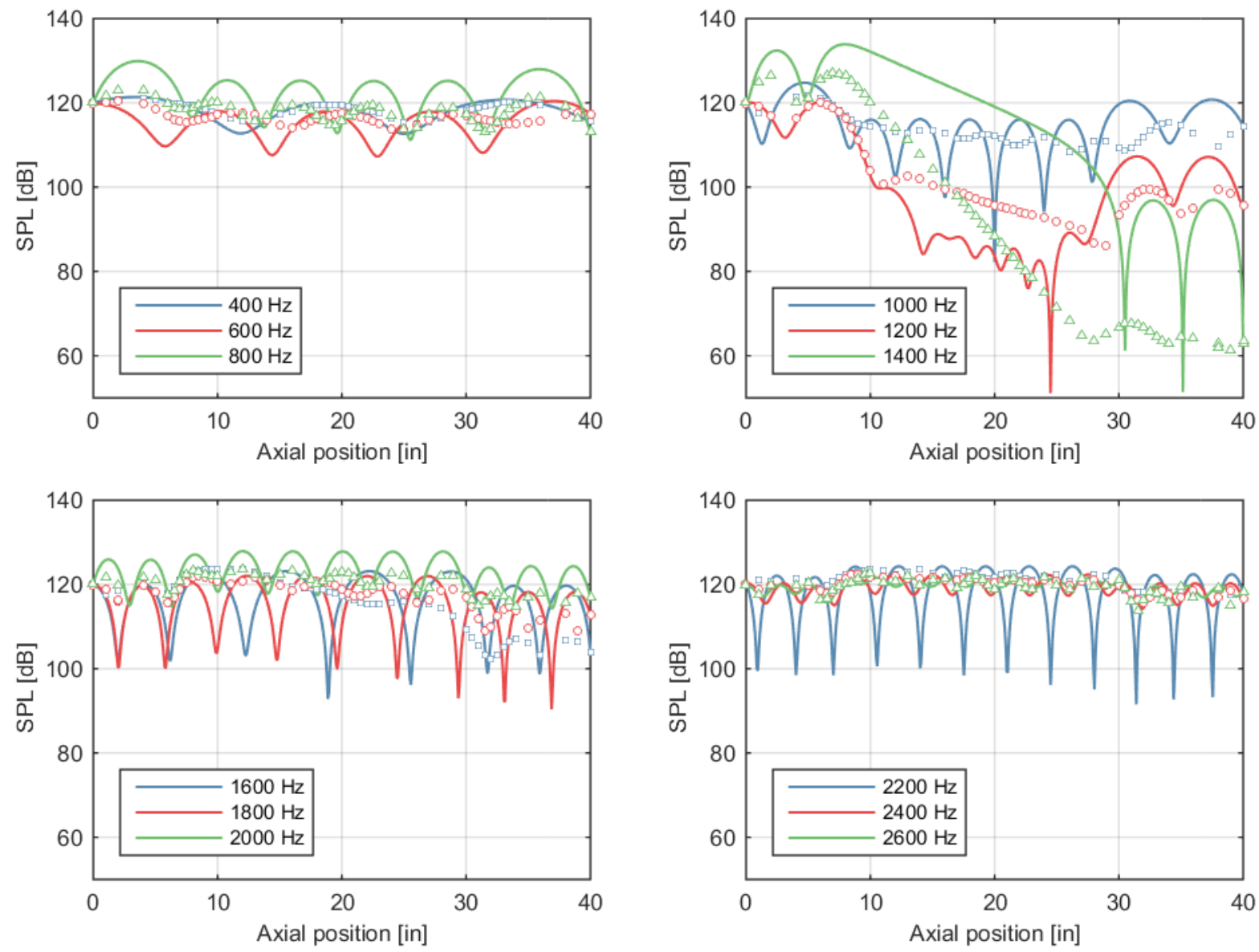

Figure 10. Sound pressure level versus position for the perforate-over-honeycomb core liner (Test 5, NBC2M000L2). GFIT measurements are indicated with markers while the solid lines correspond to predictions.

To evaluate the accuracy of the transfer impedance model, the convected Helmholtz equation impedance eduction method $^{7}$ was used to educe the impedance of the liner from the measured data collected during Test 5 . The eduction method uses an iterative process in which a finite element model (based on the convected Helmholtz equation) is solved using an estimate of the liner impedance. The predicted pressure profile in the flow duct is then compared with measurements and the assumed liner impedance is updated and the model is re-solved until the difference between predictions and measurements is within an acceptable level. Once the process has converged, the method yields an estimate of the surface impedance of the liner. Since the educed value corresponds to the surface impedance of the full liner, and not just the transfer impedance of the perforate facesheet, the reactance of the cavity (i.e., $-\cot (k D)$,

American Institute of Aeronautics and Astronautics 
where $k$ is the wavenumber and $D$ is the depth of the cavity) was removed from the measured surface reactance and then compared with the two-parameter transfer impedance prediction, as shown in Fig. 11. Note that the impedance shown in the figure has been normalized by $\rho_{0} c_{0}$. Clearly, there are significant discrepancies between the educed impedance, and the two-parameter estimate. Specifically, the resistance predicted by the model is much lower than the educed value while the predicted reactance is higher. This comparison highlights a shortcoming of the twoparameter impedance model and suggests that further work is warranted to develop a more robust impedance model for perforates. However, since this is out of the scope of the current investigation, a simple modification to the standard two-parameter model was made. Two of the semi-empirical constants were updated to better match the educed values for the $7.9 \%$ perforate for the tests without flow. Specifically, the discharge coefficient was increased by $50 \%$ from 0.771 to 1.16 , and the laminar flow friction factor was increased by a factor of 5 , from 64 to 320 . While these values are not recommended for general use, they do result in a better match with the educed impedance values for this particular facesheet, as shown in Fig. 11. Note that the updated empirical constants were only used to predict the transfer impedance of the $7.9 \%$ porosity facesheet without flow. Subsequent models with flow used the standard semiempirical constants.
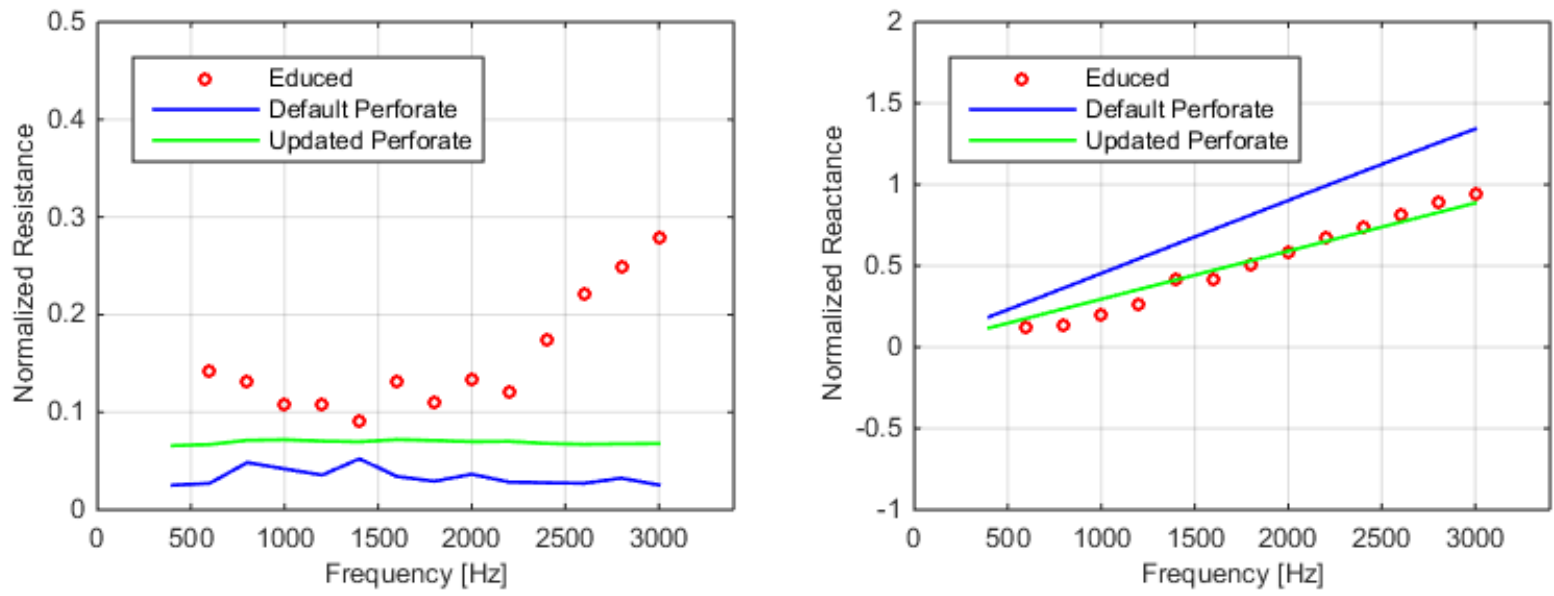

Figure 11. Normalized impedance of the perforated facesheet (with $7.9 \%$ porosity). The plot on the left is resistance (real part of impedance) and the plot on the right is reactance (imaginary part of impedance). Measurements are indicated with markers while the solid lines correspond to the semi-empirical twoparameter model. The green line corresponds to the updated model that uses nonstandard values for the discharge coefficient, $C_{D}$, and the laminar flow friction factor, $a$.

Figure 12 shows the comparison between predictions and measurements for Test 5 using the updated empirical constants for the perforated facesheet. After comparing Fig. 10 and Fig. 12, it is clear that the use of updated empirical constants results in a much better match between predictions and measurements. Therefore, the previously observed discrepancy between measurements and predictions was attributed to errors in the facesheet impedance, which is an input parameter to the model. The green bars in Fig. 6 show the new median prediction quality calculated using updated empirical constants for this perforate. As the figure shows, the update significantly improves the accuracy of the models.

American Institute of Aeronautics and Astronautics 

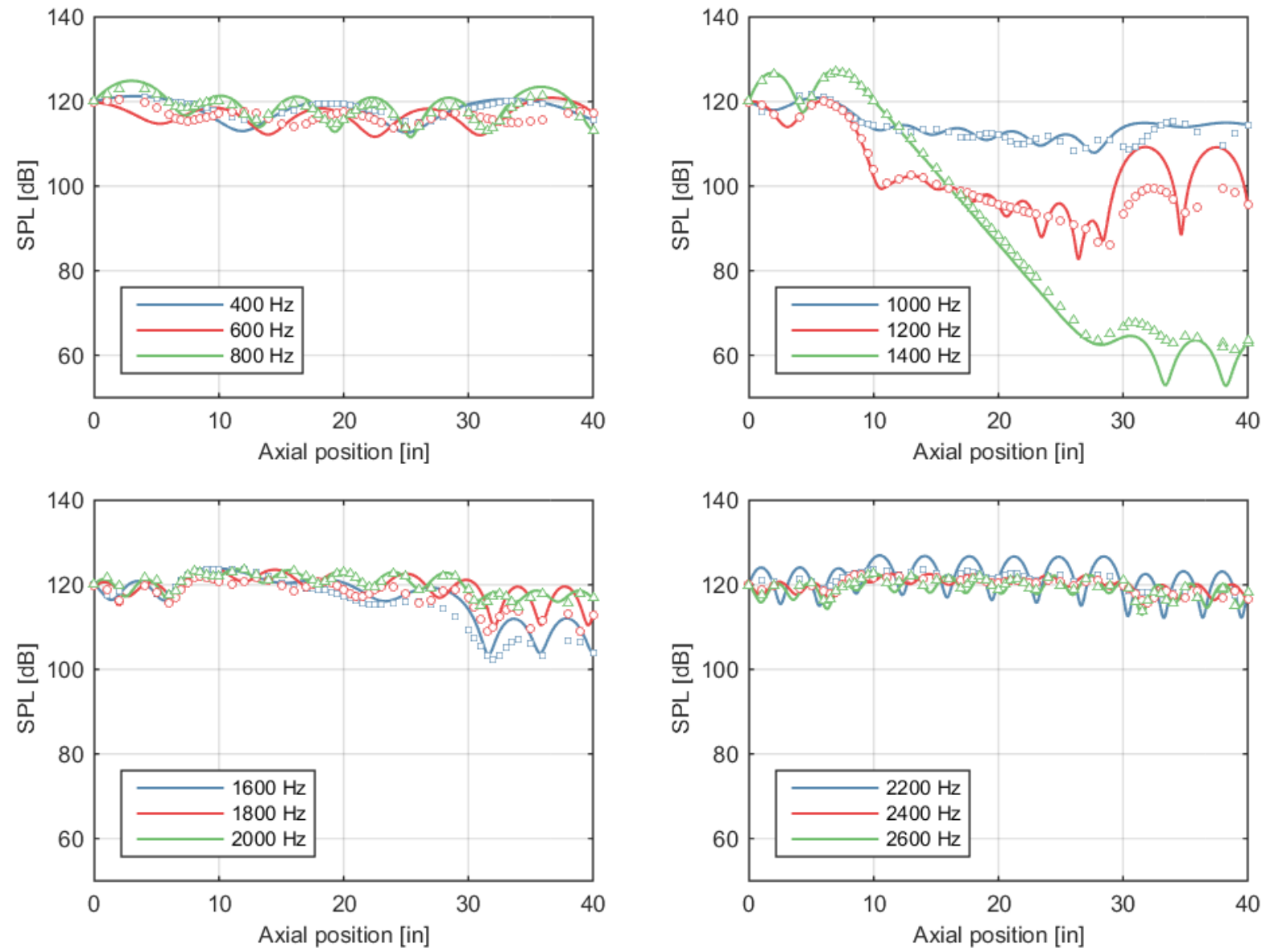

Figure 12. Sound pressure level versus position for the perforate-over-honeycomb core liner (Test 5, NBC2M000L2) using updated empirical constants for the perforated facesheet. GFIT measurements are indicated with markers while the solid lines correspond to predictions.

The same liner is considered again, but with grazing flow $\left(M_{\mathrm{C} / \mathrm{L}}=0.3\right)$. Since this test (Test 7$)$ included flow, the two-parameter model with default semi-empirical constants was used to estimate the transfer impedance of the perforated facesheet. The comparison between measurements and predictions is shown in Fig. 13. The presence of grazing flow obviously modifies the wave propagation in the flow duct, but it also modifies the resistance and reactance of the perforated facesheet. Notice that while the maximum attenuation for the no-flow case, in Fig. 12, occurred at $1400 \mathrm{~Hz}$, the maximum attenuation in Fig. 13 occurs at $1600 \mathrm{~Hz}$ and $1800 \mathrm{~Hz}$. This change is largely captured by the model, however there are noticeable discrepancies near the trailing edge of the liner, particularly at 1600, 1800, and $2000 \mathrm{~Hz}$. These discrepancies could, once again, be due to errors in the transfer impedance, since small errors in liner impedance are magnified when the attenuation is large. The discrepancies could also be attributed to shear flow, which is not captured by the model. For comparison, the median prediction quality metric is $18 \mathrm{~dB}$ for this configuration. 

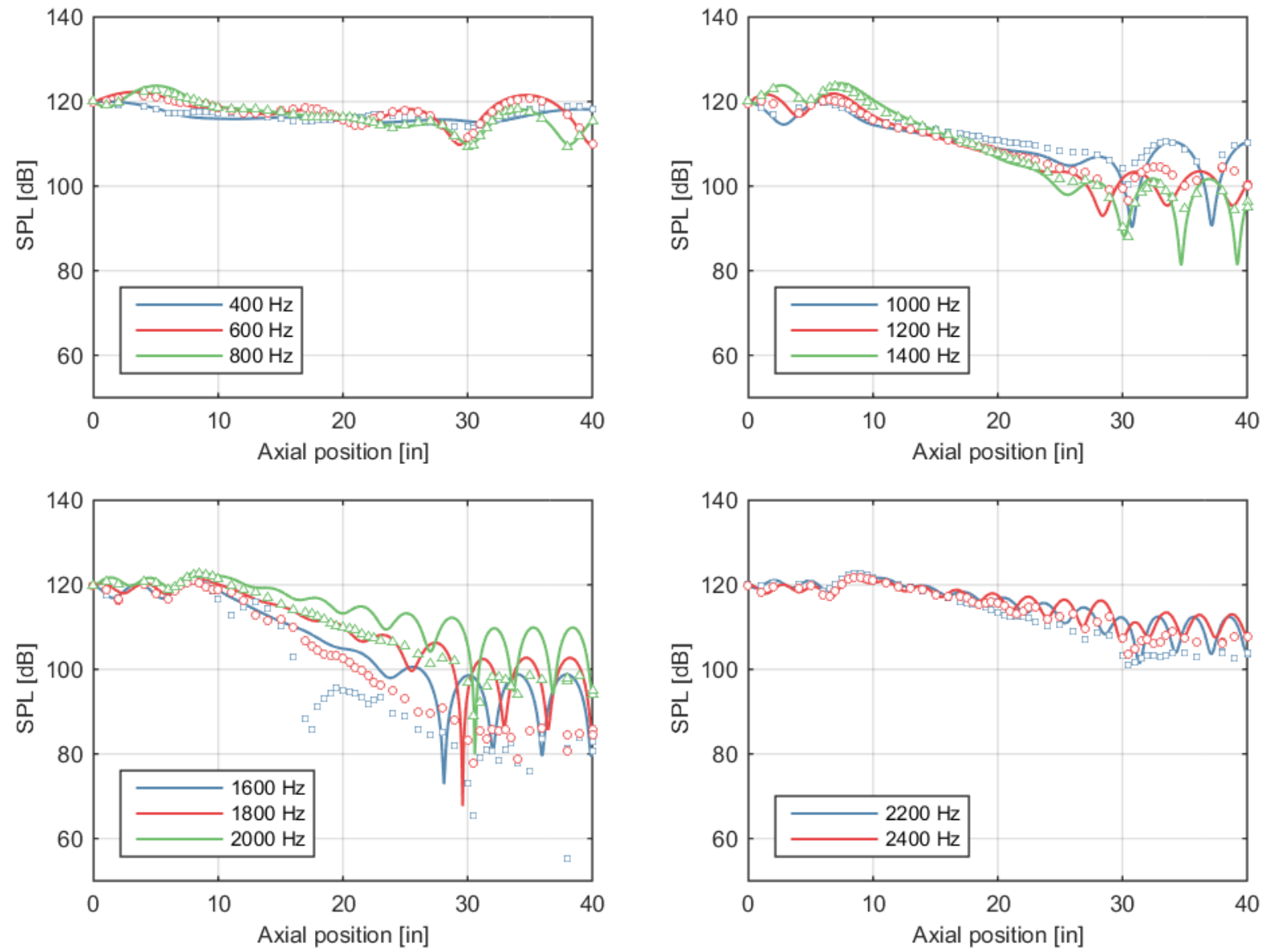

Figure 13. Sound pressure level versus position for the perforate-over-honeycomb core liner with grazing flow at $M_{\mathrm{C} / \mathrm{L}}=0.3$ (Test 7, NBC2M300L2). GFIT measurements are indicated with markers while the solid lines correspond to predictions.

In summary, the accuracy of the initial perforate-over-honeycomb predictions was relatively poor. This was attributed to inaccuracies in the transfer impedance of the perforated facesheet. Once the transfer impedance was updated, the comparison between predictions and measurements was significantly improved. This finding highlights the need for more robust impedance models for perforated facesheets. The addition of grazing flow affected the overall performance of the liner; however, this change was largely captured by the model.

\section{Perforate over Air (Test 13)}

The previous configurations were both locally-reacting liners with a conventional honeycomb core. The next configuration is an extended-reaction design without the honeycomb core, but with the same perforated facesheet. Since the core is empty it supports both transverse and lateral wave propagation, and is therefore referred to as an extended-reaction liner. The comparison for Test 13, which includes grazing flow with a centerline Mach number of 0.3, is shown in Fig. 14. In general, the model captures the major trends observed in the measurements. There are discrepancies however, particularly at $600 \mathrm{~Hz}$, which result in a relatively low median prediction quality value of $13 \mathrm{~dB}$. It is useful to note that prediction accuracy often suffers when the liner attenuation is small since standing waves dominate the response. Therefore, errors observed at those frequencies are often caused by problems with the input parameters or boundary conditions, and are not necessarily indicative of issues with the modeling tool itself.

American Institute of Aeronautics and Astronautics 

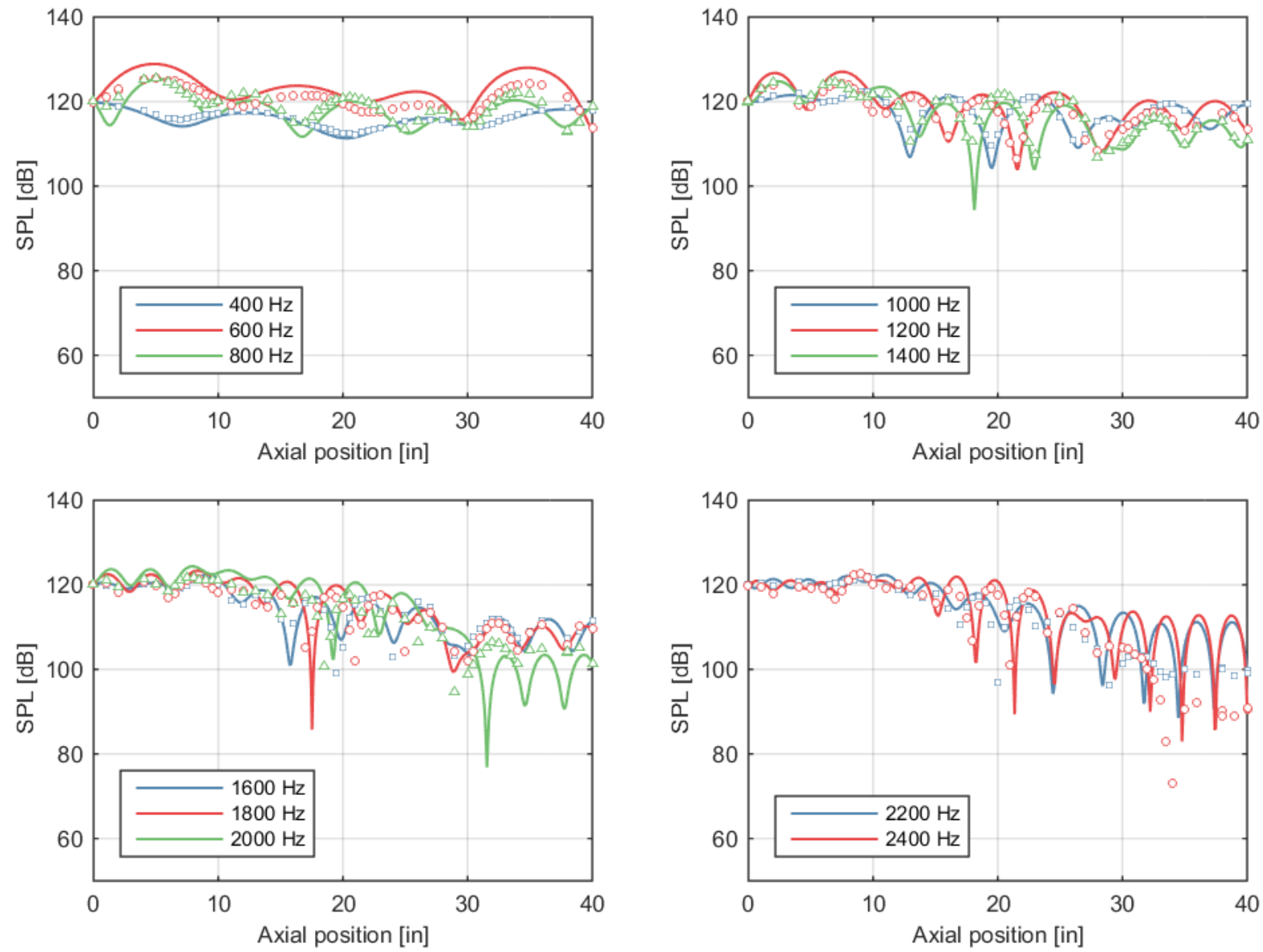

Figure 14. Sound pressure level versus position for the perforate-over-empty core liner with grazing flow at $M_{\mathrm{C} / \mathrm{L}}=\mathbf{0 . 3}$ (Test 13, NBC1M300L2). GFIT measurements are indicated with markers while the solid lines correspond to predictions.

\section{E. Bulk Material (Test 19)}

While engine liners containing bulk materials have the potential to achieve significant attenuation over a broad frequency range, they have not been widely used in commercial aircraft engines due to practical concerns. However, recent advances in materials and liner designs, along with increased demand for broadband performance, has resulted in a renewed interest in these types of liners. Therefore, the next configuration considered consists of a 2"-deep liner filled with a fibrous ceramic material and with no facesheet. The equivalent fluid properties for this material were experimentally determined using the two-thickness method described in Appendix B. The resulting effective density and speed of sound are included in Fig. 4, and are also provided in tabular form in Appendix C. The first test with a bulk material, labeled Test 19, did not include a facesheet or flow. The comparison between predictions and measurements is shown in Fig. 15. Notice that the liner gives more than $10 \mathrm{~dB}$ attenuation from the source to the termination plane at all frequencies from $400 \mathrm{~Hz}$ to $2600 \mathrm{~Hz}$. In addition, the model accurately captures the measured results and therefore the prediction quality metric is relatively high at $28 \mathrm{~dB}$ (shown in Fig. 6). Based on these results, it appears that the finite element package is able to accurately model bulk fibrous materials.

American Institute of Aeronautics and Astronautics 

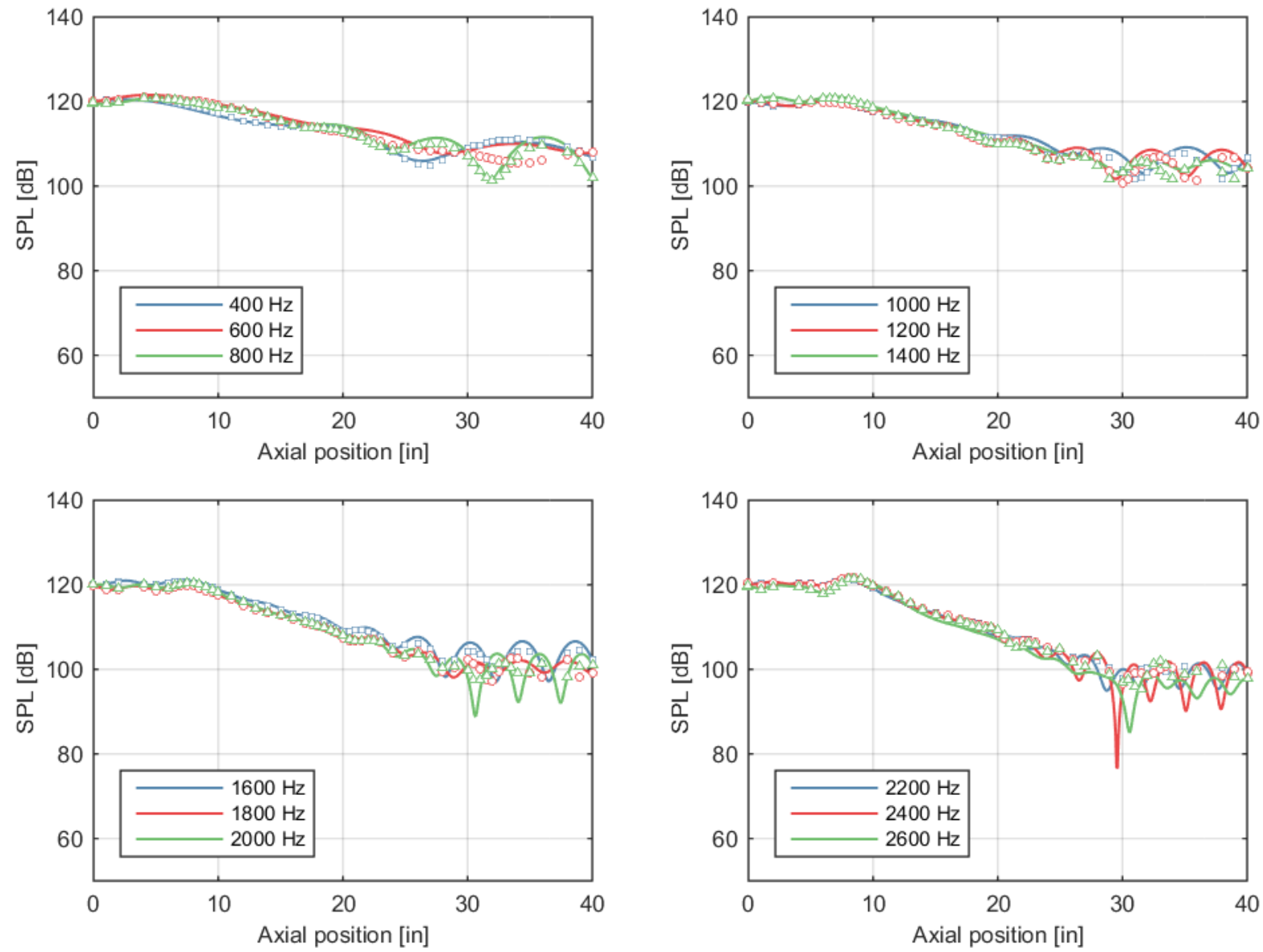

Figure 15. Sound pressure level versus position for the liner filled with ceramic fibers (Test 19, NBC5M000L2). GFIT measurements are indicated with markers while the solid lines correspond to predictions.

\section{F. Perforate over Bulk Material (Tests 23 and 29)}

The final configurations considered in this report include a perforated facesheet over the bulk material. Initially, the perforate was installed directly above the bulk material. Figure 16 compares predictions and measurements for this configuration (Test 23), which includes grazing flow with $M_{\mathrm{C} / \mathrm{L}}=0.3$. In general, the predictions closely match the measurements. A comparison of Test 13 (Fig. 14) with Test 23 (Fig. 16) shows that the addition of the bulk material improves the accuracy of the model. This is also apparent in Fig. 6, which shows that the median prediction quality improves from $13 \mathrm{~dB}$ to $19 \mathrm{~dB}$ when the bulk material is added to the core. This suggests that the addition of bulk material makes the predictions less sensitive to inaccuracies in the impedance of the perforated facesheet.

American Institute of Aeronautics and Astronautics 

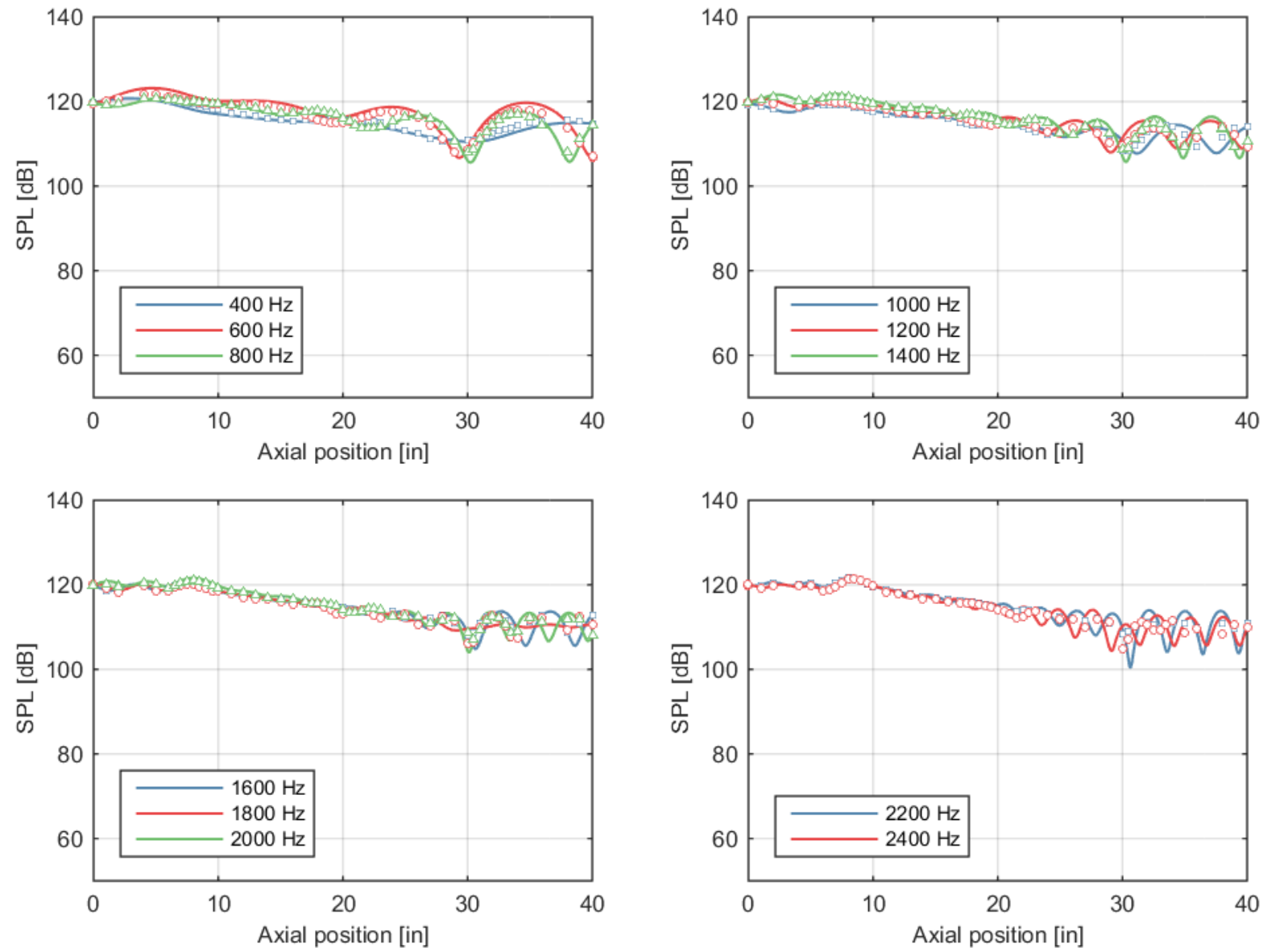

Figure 16. Sound pressure level versus position for the perforate-over-ceramic-fiber liner with grazing flow at $M_{\mathrm{C} / \mathrm{L}}=0.3$ (Test 23, NBC4M300L2). GFIT measurements are indicated with markers while the solid lines correspond to predictions.

The final configuration added an air gap between the perforate and bulk material. Specifically, Fig. 17 compares measurements and predictions for Test 29 , which includes grazing flow with $M_{\mathrm{C} / \mathrm{L}}=0.3$. Once again, the predictions capture the main trends observed in the measurements. However, the comparison is not as good as the previous configuration without an air gap, and therefore the median prediction quality metric is slightly lower (i.e., $17 \mathrm{~dB}$ instead of $19 \mathrm{~dB}$ ). The air gap in the core creates an acoustic cavity that supports lateral wave propagation with little attenuation. It is possible that the addition of the acoustic cavity within the core makes the model more sensitive to inaccuracies in the impedance of the perforated facesheet at specific frequencies, such as $600 \mathrm{~Hz}$. Regardless, the favorable comparisons between predictions and measurements indicate that the finite element package is useful for modeling engine liner concepts that include bulk materials, perforated facesheets, and grazing flow.

American Institute of Aeronautics and Astronautics 

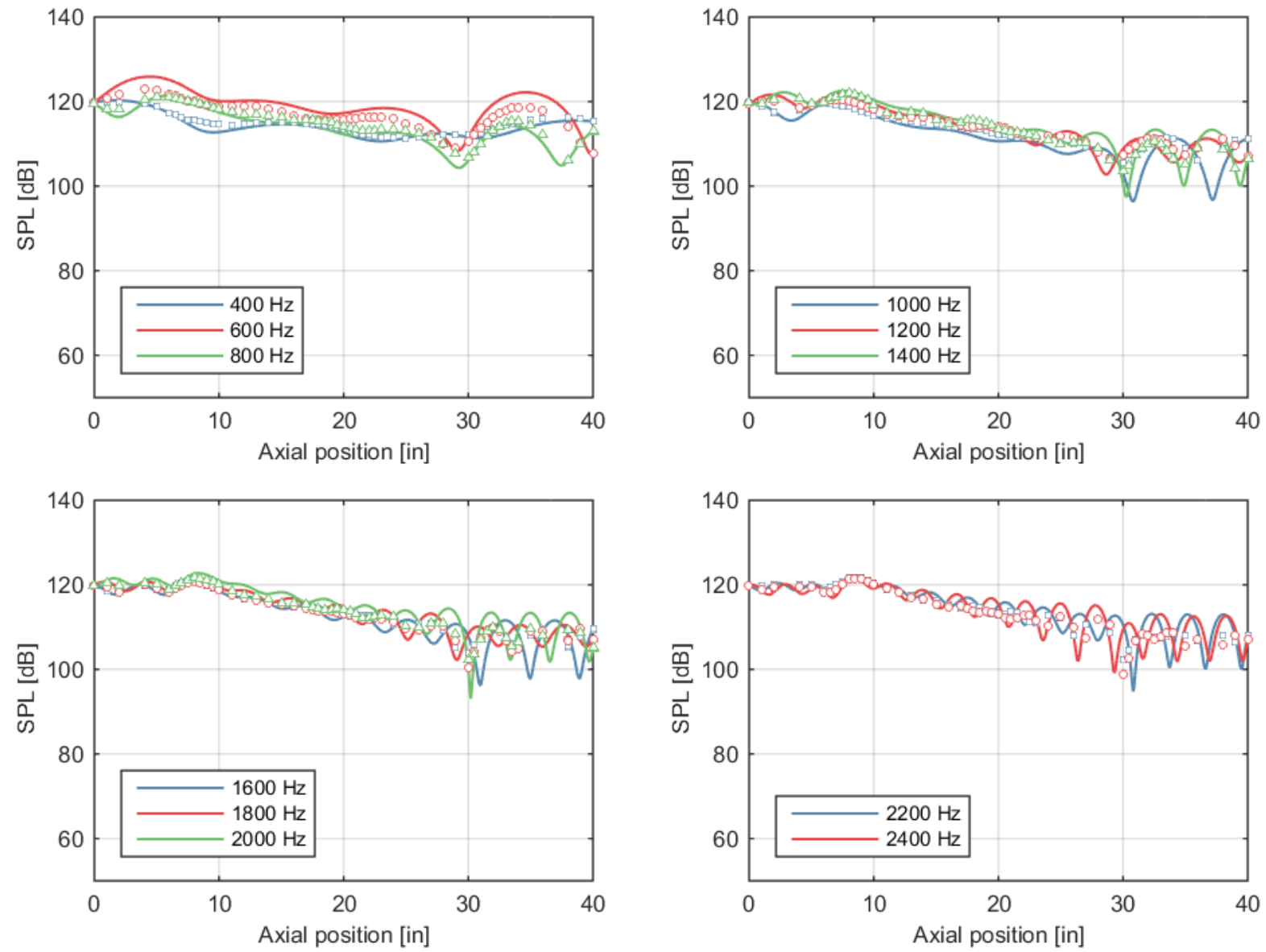

Figure 17. Sound pressure level versus position for a liner consisting of a perforate over air and ceramic fiber, with grazing flow at $M_{\mathrm{C} / \mathrm{L}}=0.3$ (Test 29, NBC3M300L2). GFIT measurements are indicated with markers while the solid lines correspond to predictions.

\section{Concluding Remarks}

The purpose of this study was to determine if COMSOL Multiphysics, a commercially-available finite element package, could be used to accurately model different types of acoustic engine liners, and in the process, collect and document a benchmark dataset that could be used in both current and future code evaluation activities. To achieve these goals, a variety of acoustic engine liner samples, ranging from conventional perforate-over-honeycomb to extended-reaction designs, were installed in one wall of the grazing flow impedance tube at the NASA Langley Research Center. The liners were then exposed to high sound pressure levels and grazing flow, and the effect of the liner on the sound field in the flow duct was measured. These measurements were then compared with predictions and the key findings are listed below:

- The comparison between predictions and measurements was excellent for the locally-reacting liner with a wire mesh over a conventional honeycomb core.

- Including grazing flow introduces small discrepancies between measurements and predictions. This may be due to the absence of the shear layer in the model.

- Initially, the accuracy of the conventional liner model that included a perforated facesheet over a honeycomb core was poor for the no-flow case. This was attributed to errors in the input parameters used to define the impedance of the perforated facesheet. After updating the impedance, the predictions closely matched measurements. This finding highlights the need for more robust impedance models for perforated facesheets.

American Institute of Aeronautics and Astronautics 
- Extended-reaction liners with an empty core can be accurately modeled using the finite element code.

- Excellent agreement was observed between predictions and measurements for the liner filled with fibrous bulk material.

- Additional comparisons demonstrated that the modeling tool is able to accurately capture the effect of liners with perforated facesheets over bulk materials.

In summary, this study demonstrated that liner models can be particularly sensitive to input parameters such as the transfer impedance of the perforated facesheet. However, when accurate input parameters are used, the finite element predictions compare very well with measurements collected using either conventional or extended-reaction liner designs. Therefore, as long as accurate input parameters are available, COMSOL should prove to be a useful addition to the current suite of acoustic propagation codes, and help enable the design of new acoustic engine liners with better broadband performance.

\section{Appendix A: Two-Parameter Model for Perforated Facesheets}

In this study, the perforated facesheet is not explicitly modeled within the finite element model. Instead, the facesheet is represented using an interior impedance condition, which corresponds to the ratio of the pressure drop across the facesheet (i.e., $p_{1}-p_{2}$ ) relative to the normal component of the particle velocity at the surface of the facesheet. The transfer impedance for the perforated facesheet was estimated using the two-parameter model. This model is discussed in detail by Ref. 5, but is summarized here for completeness.

According to the two-parameter model, the normalized impedance of the perforated facesheet can be expressed as

$$
\zeta_{f s}=\theta_{\text {lin }}+\theta_{\text {nonlin }}+\theta_{\mathrm{gf}}+i \chi_{f s}
$$

where $\theta_{\text {lin }}$ is the viscous contribution to the facesheet resistance, $\theta_{\text {nonlin }}$ is the nonlinear contribution to resistance, $\theta_{\mathrm{gf}}$ is the grazing flow contribution to resistance, and $\chi_{f_{s}}$ is the mass reactance of the facesheet. The viscous contribution is given as

$$
\theta_{\operatorname{lin}}=\frac{a \mu t}{2 \rho_{o} c_{o}\left(\sigma C_{D}\right) d^{2}}
$$

where $a$ is an empirical laminar flow friction constant with a default value of $64, \mu$ is the ambient dynamic viscosity of air, $t$ is the facesheet thickness, $\rho_{o}$ is the ambient density of air, $c_{o}$ is the ambient speed of sound of air, $\sigma$ is the open area ratio, which is equivalent to the porosity but expressed as a fraction instead of a percentage, $C_{D}$ is the discharge coefficient typically taken as 0.771 , and $d$ is the facesheet hole diameter. The nonlinear contribution is defined as

$$
\theta_{\text {nonlin }}=\frac{\kappa_{i}+\kappa_{e}}{2 c_{o}\left(\sigma C_{d}\right)^{2}} v_{\text {rms }}
$$

where $\kappa_{i}$ and $\kappa_{e}$ are empirical constants equal to 0.5 that account for entrance and exit effects, and $v_{\text {rms }}$ is the normal component of the RMS acoustic velocity at the surface of the facesheet. The grazing flow contribution to the normalized facesheet resistance is

$$
\theta_{\mathrm{gf}}=\frac{M_{\mathrm{C} / \mathrm{L}}}{\sigma\left\{2+1.256\left(\delta_{1} / d\right)\right\}}
$$

where $M_{\mathrm{C} / \mathrm{L}}$ is the free-stream (centerline) Mach number, and $\delta_{1}$ is the boundary layer displacement thickness, which is assumed to equal $1.75 \mathrm{~mm}$ for the $M_{\mathrm{C} / \mathrm{L}}=0.3$ and $M_{\mathrm{C} / \mathrm{L}}=0.5$ conditions. The normalized mass reactance of the facesheet is

$$
\chi_{f s}=\frac{k(t+\varepsilon d)}{\sigma C_{D}}
$$

where $k$ is the wavenumber and $\varepsilon$ is the hole length end correction multiplier, defined as

$$
\varepsilon=\frac{0.85(1-0.7 \sqrt{\sigma})}{1+305 M_{\mathrm{C} / \mathrm{L}}^{3}}
$$

American Institute of Aeronautics and Astronautics 
Since the impedance is a function of the normal component of the RMS acoustic velocity, an iterative process is used to determine the RMS velocity and the corresponding facesheet impedance. Specifically, an initial guess of the average RMS velocity is made and the model is solved. The RMS velocity is then updated based on the predictions, and the model is resolved. The model is assumed to be converged when the change in RMS velocity, from one solve to the next, is less than 5\%. Typically, the RMS velocity converges in 2 or 3 iterations. Note that while the impedance expressions included in this section are normalized by $\rho_{o} c_{o}$, the values entered into COMSOL are not normalized. Finally, note that while the RMS acoustic velocity varies over the length of a liner, that effect was neglected in this analysis. Instead the surface-averaged acoustic velocity was used in all impedance calculations to simplify the analysis.

\section{Appendix B: Two-Thickness Method}

For this study, the bulk materials are represented as an equivalent fluid. In other words, the material is defined using two complex-valued and frequency-dependent parameters, effective fluid density and effective speed of sound. There are several methods available to experimentally estimate equivalent fluid properties from impedance tube measurements, ${ }^{8}$ however the two-thickness method was used in this study. As the name implies, the method relies on measurements collected from samples with at least two different thicknesses. Specifically, the normalized surface impedances $\zeta_{1}$ and $\zeta_{2}$ of each sample are measured in a normal incidence impedance tube. If the second sample is twice as thick as the first (i.e., $d_{2}=2 d_{1}$ ), then the normalized characteristic specific acoustic impedance $\zeta_{c}$ and propagation constant $\Gamma$ for the material can be found as ${ }^{8}$

$$
\zeta_{c}=\sqrt{\zeta_{1}\left(2 \zeta_{2}-\zeta_{1}\right)}
$$

and

$$
\Gamma=\frac{1}{2 d_{1}} \ln \left(\frac{1+a}{1-a}\right)
$$

where $\ln ()$ denotes the natural logarithm and

$$
a=\sqrt{\frac{2 \zeta_{2}-\zeta_{1}}{\zeta_{1}}}
$$

Notice that $a$ is complex-valued and recall that the natural logarithm of a complex number is defined as

$$
\ln \left(Z e^{i \varphi}\right)=\ln (Z)+i \varphi+2 n \pi i
$$

where $Z$ is the amplitude and $\varphi$ is the phase of the complex number, and $n$ is an integer. Therefore, the solution of $\ln \left(\frac{1+a}{1-a}\right)$ in Eq. (14) is not unique and a second step is necessary to "unwrap" the imaginary component to avoid step discontinuities in the propagation constant. This is accomplished by adding multiples of $2 \pi$ to the imaginary component of $\ln \left(\frac{1+a}{1-a}\right)$ to ensure that the imaginary component of the propagation constant is positive and monotonically increasing with frequency. Once the characteristic impedance and propagation constant are found, then related quantities can be calculated, including wavenumber, complex-valued speed of sound, complex-valued density, and bulk modulus. Specifically, the relationship between the effective wavenumber and the propagation constant can be written as

$$
k_{c}=-\Gamma \mathrm{i}
$$

The effective speed of sound can be found as

$$
c_{c}=\frac{2 \pi f}{-\Gamma i}
$$

The effective density can be expressed as

$$
\rho_{c}=\frac{\zeta_{c} c \rho}{c_{c}}
$$

and the effective bulk modulus can be calculated as

$$
K_{c}=\left(\zeta_{c} c \rho\right) c_{c}
$$

American Institute of Aeronautics and Astronautics 


\section{Appendix C: Equivalent-Fluid Properties for the Bulk Materials}

Equivalent-fluid properties for the two types of bulk materials considered in this study were calculated from impedance tube measurements using the two-thickness method described in Appendix B. Although the two-thickness method was used in both cases, different facilities were used to characterize each material. Therefore, the measurement procedures will be briefly discussed before the equivalent-fluid properties for each material are presented.

The fibrous ceramic material was tested in a custom impedance tube apparatus. Specifically, measurements were collected in two different tubes with an internal diameter of 3.9" and 1.2". The larger diameter tube was used for the lower frequency range (i.e., $200-1375 \mathrm{~Hz}$ ) and the smaller diameter tube was used for high frequencies (i.e., 1000 to $6250 \mathrm{~Hz}$ ). While data was collected using 1", 2", and 3"-thick samples, the two-thickness method was performed using data from the 1" and 2"-thick samples only. Normalized specific acoustic impedance spectra for each sample were originally provided as a function of frequency in steps of approximately $4.6 \mathrm{~Hz}$. The data was then smoothed by applying a 30 point moving average. In frequency bands where data was available from both the small and large diameter tubes, the real and imaginary components of impedance were averaged separately. Another 30 point moving average was then applied to further smooth the spectra. Finally, the data was interpolated at discrete frequencies from $400 \mathrm{~Hz}$ to $3000 \mathrm{~Hz}$ in $200 \mathrm{~Hz}$ steps. The final frequency resolution was consistent with the GFIT measurements. The normalized impedances for the 1" and 2"-thick samples were then processed using the two-thickness method described in Appendix B. The resulting equivalent-fluid properties for the fibrous ceramic material are shown in Table 3.

Table 3. Complex-valued speed of sound and density for the fibrous ceramic material.

\begin{tabular}{|c|c|c|c|c|}
\hline $\begin{array}{c}\text { Frequency } \\
{[\mathbf{H z}]}\end{array}$ & $\begin{array}{c}\mathbf{R e}\left(\boldsymbol{c}_{\boldsymbol{c}}\right) \\
{[\mathbf{m} / \mathbf{s}]}\end{array}$ & $\begin{array}{c}\mathbf{I m}\left(\boldsymbol{c}_{\boldsymbol{c}}\right) \\
{[\mathbf{m} / \mathbf{s}]}\end{array}$ & $\begin{array}{c}\mathbf{R e}\left(\boldsymbol{\rho}_{\boldsymbol{c}}\right) \\
{\left[\mathbf{k g} / \mathbf{m}^{3}\right]}\end{array}$ & $\begin{array}{c}\mathbf{I m}\left(\boldsymbol{\rho}_{\boldsymbol{c}}\right) \\
{\left[\mathbf{k g} / \mathbf{m}^{3}\right]}\end{array}$ \\
\hline 400 & 62.34 & 34.02 & 11.85 & -17.93 \\
\hline 600 & 72.52 & 40.04 & 8.79 & -13.63 \\
\hline 800 & 82.73 & 45.89 & 6.93 & -10.81 \\
\hline 1000 & 91.73 & 52.91 & 5.57 & -8.93 \\
\hline 1200 & 95.91 & 58.90 & 4.50 & -8.01 \\
\hline 1400 & 92.89 & 60.00 & 3.74 & -7.70 \\
\hline 1600 & 93.42 & 57.04 & 3.61 & -7.25 \\
\hline 1800 & 102.14 & 58.75 & 3.46 & -6.47 \\
\hline 2000 & 111.62 & 57.97 & 3.32 & -5.77 \\
\hline 2200 & 119.77 & 58.06 & 3.14 & -5.16 \\
\hline 2400 & 127.12 & 58.07 & 2.99 & -4.64 \\
\hline 2600 & 135.71 & 59.27 & 2.83 & -4.19 \\
\hline 2800 & 142.85 & 60.67 & 2.72 & -3.78 \\
\hline 3000 & 149.75 & 62.27 & 2.60 & -3.46 \\
\hline
\end{tabular}

Fibrous polymer samples were tested in the NASA Langley Research Center Normal Incidence Tube (NIT). Specifically, the two-microphone $\operatorname{method}^{9,10}$ was used to compute the acoustic impedance of 0.5 ", 1 ", and 1.5"-thick samples. The tests were conducted using broadband noise with an overall SPL of $120 \mathrm{~dB}$. Since the impedance spectra was computed with a $25 \mathrm{~Hz}$ frequency resolution, the data was subsequently interpolated at discrete frequencies from $400 \mathrm{~Hz}$ to $3000 \mathrm{~Hz}$ in $200 \mathrm{~Hz}$ steps to be consistent with the GFIT measurements. The normalized impedance spectra for the 0.5 " and 1" samples were then processed using the two-thickness method described in Appendix B. The resulting equivalent-fluid properties for the fibrous polymer material are shown in Table 4.

American Institute of Aeronautics and Astronautics 
Table 4. Complex speed of sound and density for the fibrous polymer material.

\begin{tabular}{|c|c|c|c|c|}
\hline $\begin{array}{c}\text { Frequency } \\
{[\mathbf{H z}]}\end{array}$ & $\begin{array}{c}\operatorname{Re}\left(\boldsymbol{c}_{\boldsymbol{c}}\right) \\
{[\mathbf{m} / \mathbf{s}]}\end{array}$ & $\begin{array}{c}\mathbf{I m}\left(\boldsymbol{c}_{\boldsymbol{c}}\right) \\
{[\mathbf{m} / \mathbf{s}]}\end{array}$ & $\begin{array}{c}\operatorname{Re}\left(\boldsymbol{\rho}_{\boldsymbol{c}}\right) \\
{\left[\mathbf{k g} / \mathbf{m}^{3}\right]}\end{array}$ & $\begin{array}{c}\mathbf{I m}\left(\boldsymbol{\rho}_{\boldsymbol{c}}\right) \\
{\left[\mathbf{k g} / \mathbf{m}^{3}\right]}\end{array}$ \\
\hline 400 & 32.54 & 38.77 & -4.77 & -37.97 \\
\hline 600 & 39.66 & 50.46 & -3.60 & -22.86 \\
\hline 800 & 69.18 & 53.02 & 5.29 & -13.01 \\
\hline 1000 & 71.78 & 47.91 & 6.10 & -13.48 \\
\hline 1200 & 73.24 & 49.98 & 5.05 & -11.87 \\
\hline 1400 & 78.04 & 54.78 & 4.50 & -10.42 \\
\hline 1600 & 83.45 & 59.75 & 3.95 & -9.11 \\
\hline 1800 & 93.27 & 64.89 & 3.98 & -7.58 \\
\hline 2000 & 101.03 & 65.48 & 4.18 & -6.89 \\
\hline 2200 & 107.36 & 67.34 & 4.14 & -6.30 \\
\hline 2400 & 113.76 & 68.81 & 4.06 & -5.73 \\
\hline 2600 & 117.83 & 71.49 & 3.94 & -5.28 \\
\hline 2800 & 123.05 & 75.43 & 3.73 & -4.84 \\
\hline 3000 & 128.51 & 79.81 & 3.51 & -4.44 \\
\hline
\end{tabular}

\section{Acknowledgments}

The contributions of Brian Howerton, Carol Harrison, and Larry Becker are gratefully acknowledged for their role in data acquisition. The first and second authors also acknowledge the support of the NASA Advanced Air Transportation Technology Project under the Advanced Air Vehicles Program.

\section{References}

${ }^{1}$ Smith, M. J. T., Aircraft Noise, Cambridge University Press, 1989.

${ }^{2}$ Nark, D. M., Farassat, F., Pope, D. S., and Vatsa, V., "The Development of the Ducted Fan Noise Propagation and Radiation Code CDUCT-LaRC," 9h $^{\text {th }}$ AIAA/CEAS Aeroacoustics Conference, AIAA Paper 2003-3242, May 2003.

${ }^{3}$ Watson, W. R., and Nark, D. M., "Chapter 7 - Assessment of Acoustic Propagation and Radiation Codes for Locally Reacting Liners in Flow Ducts," Assessment of NASA's Aircraft Noise Prediction Capability, edited by Milo D. Dahl, NASA/TP-2012215653, July 2012, pp. 205-239.

${ }^{4}$ COMSOL, “Acoustics Module User's Guide," ver. 5.2, 2015.

${ }^{5}$ Parrott, T. L. and Jones, M. G., "Chapter 6 - Uncertainty in Acoustic Liner Impedance Measurement and Prediction," Assessment of NASA's Aircraft Noise Prediction Capability, edited by Milo D. Dahl, NASA/TP-2012-215653, July 2012, pp. 157204.

${ }^{6}$ Jones M. G., Watson, W. R., Howerton, B. M., and Busse-Gerstengarbe, S., "Effects of Mean Flow Assumption and Harmonic Distortion on Impedance Eduction Methods," AIAA Journal, Vol. 53, No. 6, 2015.

${ }^{7}$ Watson, W. R., Jones, M. G., and Parrott, T. L., "Validation of an Impedance Eduction Method in Flow," AIAA Journal, Vol. 37, No. 7, 1999.

${ }^{8}$ Smith, C. D. and Parrott, T. L., "Comparison of Three Methods for Measuring Acoustic Properties of Bulk Materials," Journal of the Acoustical Society of America, Vol. 74, No. 5, 1983.

${ }^{9}$ Chung J. Y. and Blaser, D. A., "Transfer Function Method of Measuring In-Duct Acoustic Properties. I. Theory," Journal of the Acoustical Society of America, Vol. 68, No. 3, 1980.

${ }^{10}$ Jones, M. G. and Parrott, T. L., "Evaluation of a Multi-Point Method for Determining Acoustic Impedance," Journal of Mechanical Systems and Signal Processing, Vol. 3, No. 1, 1989.

American Institute of Aeronautics and Astronautics 